\title{
Rate Effects for Mixed-Mode Fracture of Plastically Deforming, Adhesively-Bonded Structures
}

\author{
C. Sun ${ }^{\mathrm{a}}$, M. D. Thouless ${ }^{\mathrm{b}, \mathrm{c}}$, A. M. Waas, ${ }^{\mathrm{a}, \mathrm{b}}$ J. A. Schroeder ${ }^{\mathrm{d}}$ and P. D. Zavattieri ${ }^{\mathrm{d}}$ \\ ${ }^{a}$ Department of Aerospace Engineering \\ ${ }^{b}$ Department of Mechanical Engineering \\ ${ }^{c}$ Department of Materials Science and Engineering, \\ University of Michigan, Ann Arbor, MI 48109, USA \\ ${ }^{d}$ General Motors Research and Development, 30500 Mound Rd., Warren, MI 48090, USA
}

\begin{abstract}
Mixed-mode fracture of an adhesively-bonded structure made from a commercial adhesive and a dual-phase steel has been studied under different rates. Since mixedmode fracture occurs along the interface between the steel and adhesive, the cohesiveparameters for the interface were required. The mode-II interfacial properties were deduced in earlier work. In this paper the mode-I interfacial toughness and the mode-I interfacial strength were determined at different rates. The mode-I interfacial strength was not affected by rate up to crack velocities at levels associated with impact conditions, and was essentially identical to the cohesive strength appropriate for crack growth within the adhesive layer. The mode-I toughness was reduced by about $40 \%$ when the crack propagated along the interface rather than within the adhesive. Furthermore, transitions to a brittle mode of failure occurred in a stochastic fashion, and were associated with a drop in interfacial toughness by a factor of about five. The mode-I interfacial parameters were combined with the previously-determined mode-II interfacial parameters within a cohesive-zone model to analyze the mixed-mode fracture of the joints which exhibited both quasi-static and unstable fracture. The mixed-mode model and the associated cohesive parameters for both quasi-static and unstable crack propagation provide bounds for predicting the behavior of the bonded joints under various rates of loading, up to the impact conditions that could be appropriate for automotive design.
\end{abstract}

(August 15, 2008) 


\section{Introduction}

\subsection{Background and motivation}

The design of structural adhesive joints for use in automotive applications will require an ability to predict their behavior under mixed-mode loading at different rates. In particular, it will be necessary to be able to calculate the energy absorbed by a structure during a crash; this requires a design methodology that is robust when there is large-scale plasticity. For this reason, the classical tools of mixed-mode linear-elastic fracture mechanics are inappropriate. Cohesive-zone models appear to provide great potential for these applications. Whilst different numerical techniques for cohesive-zone models have been well established; there still exists a need for the development of practical techniques that can be used to extract the relevant fracture parameters under different loading conditions.

Numerical analyses using cohesive-zone models for adhesive layers bonding elastic adherends were first presented by Ungsuwarungsri and Knauss (1987), and Tvergaard and Hutchinson (1994; 1996). Yang et al. (1999) showed that it was possible to obtain two dominant cohesive parameters from mode-I experiments to characterize an adhesive layer in a plastically-deforming joint - the cohesive strength and toughness - and to use these values for quantitative predictions. It was also shown that this approach could be extended to mixed-mode loading (Yang and Thouless, 2001). Subsequent work has explored a variety of techniques to deduce cohesive parameters for adhesive joints. For example, the inverse $J$-integral technique of Li et al. (1987), has been used to determine full traction-separation curves of the adhesive layer in both mode-I (Sørensen, 2002; Sørensen et al., 2003; Andersson and Stigh, 2004) and mixed-mode conditions (Sørensen and Kirkgaard, 2006; Högberg et al., 2007). This approach has the advantage 
of giving the full traction-separation behavior in a rigorous fashion when the deformation of the system is dominated by elasticity. However, the extension of this approach to cases in which the deformation is significantly affected by plasticity, or non-linear effects in the adhesive layer, is not obvious. Alternative methods to determine the cohesive properties of the adhesive layer under these conditions often follow the general approach of Yang et al. (1999), comparing different experimental features to results of numerical calculations. This has been demonstrated in both mode-I (Ferracin et al., 2003; Andersson and Biehl, 2006) and mixed-mode conditions (Yang and Thouless, 2001; Madhusdhana and Narasimhan, 2002; Su et. al. 2004; Andersson and Biehl 2006).

Previous papers in this particular study have reported the results of investigations into mode-I and mode-II fracture in bonded joints consisting of a commercial adhesive joining dual-phase steel sheet under a range of loading rates, up to about $5 \mathrm{~m} / \mathrm{s}$. In these studies, plastic deformation of the steel always accompanied fracture. The mode-I studies (Sun et al., 2008a) exhibited crack growth that was contained completely within the adhesive layer. The mode-I properties of the adhesive layer were determined to be essentially rate-independent, except in one important regard: as the crack velocity increased, there was an increased tendency for a catastrophic transition to a relatively brittle mode of fracture. This transition was associated with a drop in the mode-I toughness from about $4.2 \mathrm{~kJ} / \mathrm{m}^{2}$ to $1.05 \mathrm{~kJ} / \mathrm{m}^{2}$ (Sun et al., 2008b). Mode-II fracture occurred along (or close to) the interface between the adhesive and steel. No transition to a brittle mode was observed in the mode-II joints, but the mode-II cohesive parameters appeared to increase slightly with rate up to crack velocities of about $2.5 \mathrm{~m} / \mathrm{s}$. The values of mode-II toughness were associated with relatively large uncertainties, but varied from 
a minimum of $8 \mathrm{~kJ} / \mathrm{m}^{2}$ to a maximum of $24 \mathrm{~kJ} / \mathrm{m}^{2}$, while the shear strength varied from about $21 \mathrm{MPa}$ to about $50 \mathrm{MPa}$ (Sun et al., 2008c).

The focus of this particular paper is to understand how the mode-I and mode-II behaviors at different rates combine under mixed-mode conditions. If adhesive-bonding is to become an integral part of automotive structural components, it will be necessary to examine the extent to which cohesive-zone models can provide robust predictions of mixed-mode failure under different loading rates, with cohesive parameters determined from a few relatively simple experiments. It is also important to understand how the fracture behavior may be modified under mixed-mode conditions. As an example, in the present case, it was demonstrated that mode-I fracture exhibited a ductile-brittle transition mode, whereas this was not observed during mode-II fracture. Conversely, quasi-static mode-II fracture showed a slight rate dependence for the cohesive parameters, while quasi-static mode-I fracture was rate independent. In addition, mode-I fracture in this system was always observed to occur within the adhesive ("cohesive" failure), but modeII loading drove the crack along one interface ("adhesive" failure). In the light of this last observation, the first task in this phase of the project was to determine appropriate values for the mode-I interfacial strength and toughness. After the interfacial mode-I properties had been deduced, the predictive nature of the model was explored by examining crack growth in two additional and independent geometries.

\subsection{Numerical model}

The modeling approach taken in this study is based on the assumption that the division between the material associated with the cohesive zone and the material associated with the continuum properties of the joint can be made at the adhesive / 
adherend interface. In other words, all the deformation of the adhesive and the energy dissipated within it is assigned to the cohesive zone. It is appreciated that this approach does not permit predictions of constraint effects that might be associated with changing the thickness or material of the adherends. However, for automotive design, it would not seem to be an undue burden to characterize an adhesive in relation to one set of materials, adhesive thickness, and sheet-metal thickness, and the approach substantially minimizes the experimental and computational complications associated with characterization and design. Furthermore, there is strong experimental evidence that accurate predictions of the performance of joint geometries can be produced with this approach (Yang and Thouless, 2001; Su et al. 2004; Andersson and Biehl, 2006).

The cohesive-zone model for mixed-mode fracture is based on that originally developed by Yang and Thouless (2001), with an adaptation for the mode-II component made necessary by the extensive shear in the present geometry (as described in Sun et al. 2008c). The mode-I and mode-II traction-separation laws are developed and described independently, but linked through a simple mixed-mode failure criterion ${ }^{1}$ :

$$
\frac{G_{I}}{\Gamma_{I}}+\frac{G_{I I}}{\Gamma_{I I}}=1
$$

where $\Gamma_{I}$ and $\Gamma_{\text {II }}$ are the mode-I and mode-II toughness, and $G_{\mathrm{I}}$ and $G_{\mathrm{II}}$ are the instantaneous values of the two corresponding energy-release rates. These energy-release rates correspond to the area under the traction-separation law at any instant in time. This mixed-mode model reproduces the conventional approaches of mixed-mode interfacial fracture mechanics (Parmigiani and Thouless, 2007) with linear-elastic adherends, but

\footnotetext{
${ }^{1}$ The model has been generalized for three-dimensional studies to include mode-III for three-dimensional studies, but this general form was not required in this part of the project.
} 
allows the effects of plasticity to be incorporated within a single fracture model. The adherends are represented in finite-element calculations by continuum elements with the appropriate constitutive properties. The adhesive layer is replaced by user-defined elements (of a thickness corresponding to the thickness of the adhesive layer) that capture the traction-separation laws the adhesive layer exerts across the interface in each mode of deformation. In the present study, the thickness of the adhesive layer was held constant, and the thickness of the adherend was also kept relatively constant, so these tractionsseparation laws were assumed to be independent of geometry, but were allowed to depend on rate.

Trapezoidal cohesive laws were used for both modes of deformation. ${ }^{2}$ The details of the mode-II law are given in Sun et al. (2008c). The form of the "adhesive" mode-I law was set equal to that of the "cohesive" mode-I law established in the prior work (Sun et al., 2008b). In particular, the initial slope was chosen to provide an approximate match to the compliance of the constrained adhesive layer, based on continuum calculations of the geometry using the constitutive properties of the adhesive. The unloading slope was chosen to be as steep as possible, while minimizing numerical instabilities. The appropriate cohesive strength and toughness for "adhesive" failure were determined by comparisons with experimental observations, as discussed in the subsequent section. Details of the numerical code for the analysis are provided in the Ph.D. thesis of Sun (2007).

\footnotetext{
2 Although details of the laws may have varying degrees of influence on some aspects of cohesive-zone calculations (Chandra et al., 2002; Li and Chandra, 2003; Li et al., 2005b, Alfano, 2006), numerical studies have confirmed that these issues do not have a significant influence on the results presented here.
} 


\section{Interfacial mode-I cohesive parameters for mixed-mode fracture}

Observations of mixed-mode fracture emphasized that the crack always grows along an interface. Mode-II fracture shows the same behavior. Therefore, the mode-II cohesive parameters deduced in earlier work from pure mode-II geometries are appropriate for use in mixed-mode analyses. However, earlier work in pure mode-I tests showed crack growth always occurring in an "cohesive" fashion, probably because of the "T"-stresses within the adhesive (Fleck et al., 1991). This means that the mode-I parameters deduced in these types of tests may not be suitable for mixed-mode analyses. Therefore, mixed-mode analyses require alternative test geometries that can be used to deduce "adhesive" values of mode-I parameters. The essential aspect of these geometries are that they are almost completely mode-I, but with enough asymmetry to drive the crack along one interface. In this paper, three geometries were used that were based on geometries of the symmetrical mode-I tests (Sun et al., 2008a; 2008b): asymmetrical tensile tests, asymmetrical double-cantilever beam tests, and asymmetric wedge tests. A wide range of loading rates was obtained by using a servo-hydraulic testing machine that could provide displacement rates of up to $250 \mathrm{~mm} / \mathrm{s}$, and a drop-tower facility that could provide displacement rates of up to $5 \mathrm{~m} / \mathrm{s}$. Details of the geometries and tests are described in the following sections.

\subsection{Asymmetric tensile tests and the cohesive strength}

The geometry for the test specimens is shown in Fig. 1a. A $2.0 \mathrm{~mm}$ thick coupon of steel (25 mm wide and $40 \mathrm{~mm}$ long) was joined to a $1.4 \mathrm{~mm}$ coupon of steel by a $5 \mathrm{~mm}$ long bond of adhesive. The bonding process was identical to that described in the earlier studies, and the thickness of the adhesive layer was maintained at $0.8 \pm 0.2 \mathrm{~mm}$ by 
the use of uniform-sized glass beads as spacers. Teflon tape was put on the thinner side of the steel arm to trigger crack propagation along the interface with the thinner side, since the sense of the asymmetry was to drive the crack along that interface. Thick steel tabs were attached to the ends of the bonded coupons, so that a load could be applied through a pin joint. This design allowed a relatively uniform tensile load to be applied across the bond, but left the adhesive constrained only by the unsupported sheet metal.

Four tests were performed at a displacement rate of $0.01 \mathrm{~mm} / \mathrm{s}$, and four tests were performed at a displacement rate of $100 \mathrm{~mm} / \mathrm{s}$. These displacement rates corresponded to nominal strain rates of about $0.0125 / \mathrm{s}$ and $125 / \mathrm{s}$ across the adhesive. Optical observations after the tests showed that the crack propagation was "interfacial" (or "adhesive") in the sense that most of the adhesive was left on the thicker arm. The nominal strength of the joint, defined as the peak load divided by the load-bearing area of the adhesive was constant for all tests, and equal to $23 \pm 2 \mathrm{MPa}$. There was no difference in the nominal strength at the two strain rates, even though the rates were separated by four orders of magnitude. This observation was consistent with the previous observations for the symmetrical tensile test (Sun et al., 2008b).

Numerical simulations of this geometry were performed using the full range of the mode-II cohesive parameters appropriate for this system (Sun et al., 2008c). The mode-II toughness was varied between $8 \mathrm{kJm}^{-2}$ and $24 \mathrm{kJm}^{-2}$, and the cohesive shear strength was varied between $20 \mathrm{MPa}$ and $50 \mathrm{MPa}$, representing the full range of the effects of the loading rate. Different values of the interfacial mode-I strength and toughness were used in the calculations to explore how the nominal strength of the joint varied with the cohesive parameters for this system. These calculations indicated that the 
nominal strength depended only on the mode-I cohesive strength of the interface, for what was considered to be a reasonable range of values for the interfacial toughness. Furthermore, the calculations showed that the stress in the cohesive zone was essentially uniform along the bond, which meant that the nominal strength of the joint was identical to the interfacial strength. Therefore, it was deduced that the mode-I interfacial strength, over many orders of magnitude for the loading rate, was $\hat{\sigma}_{i}=23 \pm 2 \mathrm{MPa}$. This is equal to the mode-I cohesive strength of $24 \pm 3 \mathrm{MPa}$ that had been obtained earlier for the adhesive layer (Sun et al., 2008b). In other words, the fact that fracture occurred along the interface, rather than through the middle of the adhesive, did not seem to affect the strength parameter.

\subsection{Asymmetrical double-cantilever beam geometry and the quasi-static toughness}

Bonded, asymmetrical, double-cantilever beams (ADCB) were used to determine the mode-I, quasi-static, interfacial toughness. The geometry of these specimens is shown in Fig. 1b, and a micrograph taken during a test is shown in Fig. 2. The specimens were tested with a servo-hydraulic testing machine. Four tests were conducted at a crosshead displacement rate of $1 \mathrm{~mm} / \mathrm{s}$, two tests at a rate of $10 \mathrm{~mm} / \mathrm{s}$, and three tests at a rate of $200 \mathrm{~mm} / \mathrm{s}$. In all cases, fracture occurred along the interface between the adhesive and steel adherend. Transitions to dynamic fracture were observed for the tests conducted at the intermediate velocities, but they weren't observed in the tests done at the slowest and fastest rates. Quasi-static growth occurred at velocities of $0.75 \mathrm{~mm} / \mathrm{s}$ and $150 \mathrm{~mm} / \mathrm{s}$ without transitions to dynamic fracture, but transitions to dynamic failure were observed at crack velocities of $7.5 \mathrm{~mm} / \mathrm{s}$. As with the symmetrical mode-I studies, these results 
emphasize the stochastic nature of the transitions to dynamic fracture, and the absence of a critical crack velocity for the onset of instability.

Representative load-displacement plots for the quasi-static results are shown in Fig. 3, along with plots for experiments in which transitions to dynamic fracture occurred. The associated plots of crack length versus cross-head displacement are shown in Fig. 4. In these experiments, dynamic fracture occasionally occurred before any quasistatic crack growth had been observed, In this regard, the results were different from those associated with the perfectly symmetrical DCB experiments in which the first onset of dynamic fracture always occurred after an initial period of quasi-static crack growth. However, even in the present set of experiments all subsequent occurrences of dynamic fracture occurred as transitions from a quasi-static mode of crack growth. This indicates that this behavior may simply reflect the random nature of the transition, and is not inconsistent with the qualitative understanding of the transition proposed in Sun et al. (2008b). Furthermore, while no quasi-static crack growth was detected before the first onset of dynamic fracture, inspection of the failure surface after fracture (Fig. 5) indicated a region at the initial crack tip which exhibited the "whitening" associated with quasi-static crack growth. This suggests that the toughening mechanisms associated with quasi-static fracture may have been triggered before the transition to dynamic fracture, even in the absence of quantifiable crack growth. A related observation was made in the fracture of rubber-toughened epoxies at high loading rates by Du et al., 2000, and is again consistent with the qualitative model presented in Sun et al. (2008b).

Numerical simulations for the quasi-static tests with this geometry were performed using the full range of cohesive parameters appropriate for the mode-II 
cohesive law (Sun et al., 2008c). Different values of the interfacial mode-I strength and toughness were used in the cohesive-zone model to compute load-displacement curves, which were then compared to the experimental results. However, these calculations showed that the load-displacement curves for the particular geometry and materials used in this study were sensitive only to the mode-I interfacial toughness. Therefore, while the asymmetrical tensile tests provided a value for the interfacial strength, the asymmetric double-cantilever beam tests provided a value for the interfacial toughness. As shown in Fig. 6, excellent fits to the quasi-static test load-displacement curves could be obtained using an interfacial mode-I toughness of $\Gamma_{\mathrm{Ii}}=2.8 \pm 0.3 \mathrm{~kJ} / \mathrm{m}^{2}$. This range of toughness also provides consistent fits between the numerical and experimental results for the variation of the crack length with cross-head displacement (Fig. 7).

Summaries of the sets of interfacial cohesive parameters that provide good numerical fits to both experiments described above are shown in Fig. 8. The intersection between the two sets indicates that the interfacial normal cohesive strength is $\hat{\sigma}_{i}=23 \pm 2 \mathrm{MPa}$, while the quasi-static interfacial mode-I toughness is $\Gamma_{\mathrm{Ii}}=2.8 \pm 0.3 \mathrm{~kJ} / \mathrm{m}^{2}$, over the full range of rates explored in these tests. ${ }^{3}$ This figure is identical in form to a similar plot for the symmetrical mode-I tests (Sun et al., 2008b). A comparison between the two sets of results indicates that the mode-I strength was not affected by the locus of failure, but the quasi-static mode-I toughness dropped from a value of $4.2 \pm 0.3 \mathrm{~kJ} / \mathrm{m}^{2}$ to $2.8 \pm 0.3 \mathrm{~kJ} / \mathrm{m}^{2}$ when the crack grew along the interface, rather than in the middle of the adhesive layer. Finally, it is noted that the computed ratio of $\left(G_{\mathrm{I}} / \Gamma_{\mathrm{II}}\right) /\left(G_{\mathrm{II}} / \Gamma_{\mathrm{IIi}}\right)$ at the crack tip at fracture for the ADCB is equal to $0.95 / 0.05$, while the

\footnotetext{
${ }^{3}$ Numerical calculations confirmed that the strain rates in the adhesive layer during the ADCB tests were within the range of strain rates obtained with the asymmetrical tensile tests.
} 
ratio for the asymmetric tensile test is $0.96 / 0.04$, indicating that both systems are dominated by mode-I deformation, and are, therefore, appropriate for the present analysis.

\subsection{Asymmetric wedge geometry and the toughness of the brittle mode}

Asymmetrical wedge tests permitted higher crack velocities to be explored than could be obtained from the ADCB tests. The geometry of the specimens used for these tests is shown in Fig. 9. The bonded coupons were forced over a stationary wedge by means of a mass of $40 \mathrm{~kg}$ dropped from heights between $50 \mathrm{~mm}$ and $1000 \mathrm{~mm}$. The wedge had an angle of $10^{\circ}$, a tip diameter of $1 \mathrm{~mm}$, and a length of $120 \mathrm{~mm}$. The sides of the specimens were painted white, with a scale scribed onto them. A high-speed camera ${ }^{4}$ was focused on the wedge; the crack and specimen velocities were monitored by comparing the positions of the wedge tip and crack tip relative to the scale. Micrographs taken during the tests, and micrographs of the specimens after fracture, clearly showed that the crack always propagated along the interface between the adhesive and the steel. Two specimens, tested at different rates exhibited only quasi-static crack growth; the others exhibited random transitions to dynamic fracture. In all cases, the thin arm of the joint deformed into an arc of a circle, while the thicker arm remained straight (Fig. 10). The radius of curvature of the deformed arm was $82 \pm 8 \mathrm{~mm}$ for both of the tests that exhibited quasi-static crack growth.

The displacements of the specimen relative to the wedge are plotted against time in Fig. 11. From these plots it will be observed that the average specimen velocities (and, hence, average crack velocity) ranged between about $950 \mathrm{~mm} / \mathrm{s}$ and $4400 \mathrm{~mm} / \mathrm{s}$, and that

\footnotetext{
${ }^{4}$ A Kodak EktaPro Motion Analyzer, Model 1000HRC. This system can capture a maximum of 2728 frames at a maximum framing rate of 1000 full frames per second.
} 
the velocities remained relatively constant for the duration of all the tests. Plots of crack extension versus time after impact are shown in Fig. 12. These plots show that two tests conducted with drop heights of $50 \mathrm{~mm}$ and $200 \mathrm{~mm}$, exhibited quasi-static crack growth at average velocities of about $950 \mathrm{~mm} / \mathrm{s}$ and $2000 \mathrm{~mm} / \mathrm{s}$. The other three tests, conducted with drop heights of $50 \mathrm{~mm}, 200 \mathrm{~mm}$ and $1000 \mathrm{~mm}$ exhibited interfacial "stick-slip" behavior with quasi-static crack growth being punctuated by occasional transitions to dynamic fracture. There was a considerable range of crack velocities for the quasi-static mode after transitions to dynamic fracture occurred, but it should be noted that quasistatic crack growth did occur at a velocity very comparable to the average specimen velocity, even at the fastest rate. It was noted that transitions to dynamic fracture were not as prevalent for these mode-I "adhesive" failures at the interface as they had been for mode-I "cohesive" crack growth within the adhesive layer (Sun et al., 2008a). Figure 13, shows that when the crack was growing in a quasi-static mode, the distance between the crack tip and the wedge was in the range of 6 to $10 \mathrm{~mm}$ with no correlation to crack velocity (outside specimen-to-specimen variation), but increased to between 16 and $24 \mathrm{~mm}$ immediately after a transition to dynamic fracture.

A plane-strain, static ABAQUS/Standard 2D analysis with mixed-mode cohesive elements along the interface was established to model the asymmetric wedge tests. As with the previous simulations, the full range of the mode-II cohesive parameters determined in Sun et al. (2008c) was used, and the interfacial mode-I strength was fixed at $23 \pm 2 \mathrm{MPa}$. Owing to the fact that the specimens rotated during the test, so that the thick arm with the adhesive layer lay parallel to one of the wedge surfaces (Fig. 10), only the thin arm was modeled in the simulations. The surfaces of the wedge and the thin arm 
were defined as contact surfaces. The adhesive layer was modeled by mixed-mode cohesive-elements of thickness $0.8 \mathrm{~mm}$. One edge of these elements was attached to a rigid boundary, representing the interface between the adhesive and the thicker arm. The other edge of these elements was attached to the thinner arm. The wedge was advanced through the specimen, with one surface of the wedge being constrained to the plane that defined the initial boundary between the adhesive and thinner arm (Fig. 14). Crack advance and deformation evolved naturally as the finite-element calculations proceeded. In these calculations, only the crack length and curvature of the thinner arm were considered to be of interest. Therefore, frictional effects were not included in this model, because our prior studies have confirmed that friction does not affect these two parameters, since the wedge was sharp enough for the transverse shear forces that opened the crack to be dominated by the normal reaction force (not the frictional force). ${ }^{5}$

When the upper bounds for the quasi-static, mode-I, interfacial toughness $\left(\Gamma_{\mathrm{Ii}}=3.1 \mathrm{~kJ} / \mathrm{m}^{2}\right)$ were used in the analysis, the crack was calculated to grow at a fixed distance of $8 \mathrm{~mm}$ ahead of the wedge tip. When the lower bounds for the quasi-static, mode-I, interfacial toughness $\left(\Gamma_{\mathrm{Ii}}=2.5 \mathrm{~kJ} / \mathrm{m}^{2}\right)$ were used, the crack was calculated to grow at a fixed distance of $6 \mathrm{~mm}$ ahead of the wedge tip. These results were not sensitive to the mode-II cohesive parameters, and were reasonably consistent with the experimentally observed values. Furthermore, the radius of curvature for the thinner arm was calculated to be $79 \pm 8 \mathrm{~mm}$ for the range of interfacial cohesive parameters. Again, this was consistent with the observed value of $82 \pm 8 \mathrm{~mm}$. In these calculations, the

\footnotetext{
${ }^{5}$ As discussed in a companion paper (Sun et al., 2008b), the force pushing the wedge along the interface, and the energy dissipated, is sensitive to friction. When that force, or the total energy dissipated, is of interest, friction must be included in the calculations, because they can be dominated by frictional effects.
} 
crack-tip ratio of $\left(G_{\mathrm{I}} / \Gamma_{\mathrm{Ii}}\right) /\left(G_{\mathrm{II}} / \Gamma_{\mathrm{II}}\right)$ was computed to be $0.94 / 0.06$ at fracture, confirming that crack growth in the asymmetric wedge test geometry was dominated by mode-I deformation, and insensitive to the mode-II cohesive parameters.

The mode-I interfacial toughness for the brittle mode of fracture was not calculated from the ADCB geometry, and was left as an unknown parameter to be determined from the wedge tests. The toughness was deduced by repeating the numerical calculations with different values of toughness until the computed distance of the crack tip ahead of the wedge matched the experimental observations for how far the crack jumped ahead of the wedge after transition to a brittle mode of fracture. Agreement was found when the toughness was reduced to a range of between $0.51 \mathrm{~kJ} / \mathrm{m}^{2}$ and $0.65 \mathrm{~kJ} / \mathrm{m}^{2}$. Therefore, the interfacial mode-I toughness for the brittle mode of fracture was taken to be $0.58 \pm 0.07 \mathrm{~kJ} / \mathrm{m}^{2}$. As a comparison, the earlier work (Sun et al., 2008b) showed that the mode-I toughness for the brittle mode was about $1 \mathrm{~kJ} / \mathrm{m}^{2}$ when the crack grew "cohesively" within the adhesive; for both the toughened and brittle modes of crack growth, the mode-I toughness was reduced by about $40 \%$ when the locus of fracture moved to the interface. A summary of all the mode-I and mode-II cohesive parameters appropriate for mixed-mode fracture in the adhesive/adherend system used in this study is given in Table 1.

\subsection{Stick-slip in the asymmetric-double-cantilever-beam geometry}

As described in Sun et al. (2008b) either the double-cantilever-beam geometry or the wedge geometry can be used to deduce the mode-I toughness for this system, with the other geometry being used to verify the values. In the earlier work on mode-I "cohesive" failure, the toughness for both the brittle and toughened modes of crack growth were 
deduced from the DCB geometry and verified using the wedge geometry. In the present work on "adhesive" failure, the toughness of the toughened mode was determined from the ADCB geometry and verified by the wedge geometry, but the toughness of the brittle mode was determined from the wedge geometry. In this section, the value of toughness is verified by using it to analyze stick-slip behavior in the ADCB geometry.

The value of the lower-bound mode-I toughness can be validated by using it in a numerical model of the ADCB test, and comparing the distance that the crack jumps after the transition to the observed distance. In this regard, it should be noted that the jump distance is controlled by the displacement and the deformation of the specimen at the onset of dynamic fracture, and the value of toughness for the brittle mode. If there is a quantifiable extent of quasi-static crack growth, the first two of these immediately evolve from a cohesive-zone analysis with toughened elements placed over an appropriate length of the interface. ${ }^{6}$ In the present case, there was no evidence of quasi-static crack growth before the first transition to dynamic, but it appears that the toughening mechanism had been triggered before this event. ${ }^{7}$ Therefore, the length of interface over which to place the initial block of toughened elements had to be deduced by matching the load at which the transition to dynamic crack growth was calculated to that observed. Thus, the load for the transition, rather than the extent of quasi-static crack growth, was used as an additional input parameter. Once this was established, the crack length at which the dynamic crack growth was arrested was dependent only on the value of the mode-I

\footnotetext{
${ }^{6}$ This is the approach that was taken in Sun et al. (2008b). Owing to the stochastic nature of the transition, the onset of dynamic fracture cannot be modeled a priori.

${ }^{7}$ From the perspective of a cohesive zone model, crack growth does not occur until the full tractionseparation curve has been traversed. Therefore, it is possible for a region ahead of the crack tip to start following the traction-separation law corresponding to the toughened mode, but to have the material ahead of this region fail in a brittle mode before the crack starts to grow.
} 
toughness; a comparison of this length then becomes a means of validating the value that is independent of the test used to deduce it.

The calculations were done using a plane-strain, dynamic ABAQUS/Standard 2D model. A small region of tough elements $\left(\Gamma_{\mathrm{I} i}=2.8 \pm 0.3 \mathrm{~kJ} / \mathrm{m}^{2}\right)$ were placed ahead of the initial tip of the crack in a numerical model of the ADCB geometry of Fig. 1a. The rest of the interface was modeled by brittle elements $\left(\Gamma_{\mathrm{I} i}{ }^{\mathrm{d}}=0.58 \pm 0.07 \mathrm{~kJ} / \mathrm{m}^{2}\right)$. The appropriate length for this initial region of tough elements was unknown at the onset of the calculations. By trial and error, it was observed that the numerical value for the peak load experienced by the ADCB geometry could be matched to the experimental values if these tough elements were limited to only $0.5 \mathrm{~mm}$ from the initial crack tip.

The calculations were then done with tough elements placed over this initial region, and brittle elements over the rest of the interface. After the transition to dynamic fracture, the crack jumped to $7.5 \pm 1.5 \mathrm{~mm}$ (corresponding to the range of uncertainty in the mode-I toughness). This matched the experimental results of $7 \pm 1 \mathrm{~mm}$, and provided evidence that the value of the interfacial mode-I dynamic toughness was correct. It should be noted that in this analysis, none of the toughened elements reached the failure criterion before the onset of dynamic fracture; in other words no quasi-static crack growth occurred before the transition. The transition to dynamic failure occurred when the brittle elements placed immediately after the tough elements failed. At this point, the crack became unstable, jumping backwards to join the main crack and forwards to the new arrest position. This mimicked what appeared to be happening experimentally, and supports the notion that the toughening mechanism is triggered at the crack tip, but is not fully developed along what would be the whole length of the process zone for steady- 
state toughened crack growth. This is consistent with a qualitative understanding of the transition presented in Sun et al. (2008b) in which the stresses for cleavage and toughening are comparable, and there is a slightly rate-dependent but random aspect to whether toughening will be triggered in any particular element of the material.

\section{A mixed-mode single-lap shear geometry}

A single-lap shear geometry was used as example of a mixed-mode configuration to validate the mixed-mode cohesive properties. It should be noted that transitions to the dynamic mode of fracture appear to be stochastic in nature, and can't be predicted by the numerical models. At this stage, the models can only be used to predict upper and lower bounds for the strengths and energy-absorbing capabilities of different joints, or used for calculations where the type of crack growth is known a priori. The geometry of the specimens is shown in Fig. 15. The experiments were performed using a servo-hydraulic machine at constant displacement rates of $0.1 \mathrm{~mm} / \mathrm{s}, 10 \mathrm{~mm} / \mathrm{s}$, and $200 \mathrm{~mm} / \mathrm{s}$. The experimental load-displacement results are shown in Fig. 16. The displacements associated with the experimental results were obtained from the cross-head displacements, as calibrated from a simple tensile test performed on an equivalent steel coupon. The cracks grew from both ends of the sample, but not necessarily in a completely symmetrical fashion. Once the cracks started to propagate, they accelerated very rapidly to cause failure of the interface. The range of applied displacement over which the cracks propagated was so small that the crack velocity could not be measured, nor was it possible to deduce whether there was a transition to dynamic fracture. However, observations of the fracture surfaces after the tests suggested that only quasistatic crack growth had occurred. 
The cohesive-zone analysis of this test was implemented within a static ABAQUS/Standard 2D plane-stress model, with cohesive elements along the entire interface. Two sets of calculations were done: one with the cohesive parameters set to the range appropriate for interfacial toughened fracture $\left(\Gamma_{\mathrm{I}}=2.5 \sim 3.1 \mathrm{~kJ} / \mathrm{m}^{2}\right.$, $\Gamma_{\mathrm{II}}=8 \sim 23 \mathrm{~kJ} / \mathrm{m}^{2}$ ), and one with the parameters appropriate for brittle fracture $\left(\Gamma_{\mathrm{Ii}}{ }^{\mathrm{d}}=0.51 \sim 0.65 \mathrm{~kJ} / \mathrm{m}^{2}, \Gamma_{\mathrm{II}}=8 \sim 23 \mathrm{~kJ} / \mathrm{m}^{2}\right)$. The resulting numerical load-displacement plots have been added to Fig. 16. As can be seen from that figure, there is a satisfactory agreement between the experimental results and the numerical results, with the numerical results for the fully-toughened mode of crack growth bracketing the experimental results. There is a fairly wide bound on the predictions for the total displacements that can be accommodated by the joints, but there is a similarly broad range observed experimentally. The numerical results indicated that crack growth occurred only during the last millimeter of applied displacement. Again, this result is consistent with what was observed experimentally. The mode-mixedness of this configuration, as defined by the ratio of $\left(G_{\mathrm{I}} / \Gamma_{\mathrm{I}}\right) /\left(G_{\mathrm{II}} / \Gamma_{\mathrm{II}}\right)$ at the crack tip at fracture, was about $0.2 / 0.8$. This confirms that this geometry provided an intermediate level of mode-mixedness, distinct from the mode-I and mode-II geometries used to determine the cohesive parameters. Therefore, the levels of agreement between the numerical and experimental results do provide some validation of the predictive nature of the model.

\section{Conclusions}

Under mixed-mode conditions, a crack will grow along the interface of a joint. The mode-I cohesive parameters appropriate for analyzing these conditions can be determined by designing geometries that have just enough asymmetry to drive the crack 
to the interface, but are dominated by the mode-I behavior of the bonding. In the present paper, "adhesive" mode-I cohesive parameters were determined for a system consisting of a commercial rubber-toughened epoxy and a dual-phase steel, and compared to the values obtained previously for "cohesive" mode-I parameters. In this particular case, the mode-I strength did not appear to depend on the crack path, but the toughness was reduced by about $40 \%$ when the crack propagated along the interface, rather than through the adhesive. Random transitions to a brittle and dynamic mode of fracture were observed in mode-I fracture for both trajectories. In each case, these transitions were associated with a reduction in the toughness by a factor of about five. While the probability of a transition to the brittle mode of fracture appeared to depend on the loading rate in both cases, there seemed to be no effects of rate on the mode-I cohesive parameters.

The stochastic nature of the transitions between the dynamic and quasi-static modes of crack growth means that numerical models of rate-dependent fracture may have to be limited to analyzing upper and lower bounds for the performance of automotive joints under crash applications (or as a means of validating different adhesive/adherend systems or geometries). An example of a mixed-mode cohesive-zone analysis combining the mode-I and mode-II cohesive parameters measured independently demonstrated the ability of the model to provide good bounds for the performance of a lap-shear geometry.

\section{Acknowledgements}

C. Sun, M. D. Thouless and A. M. Waas gratefully acknowledge the financial support of General Motors. 


\section{References}

Alfano, G. "On the influence of the shape of the interface law on the application of cohesive-zone models," Composites Science and Technology, 66, 723-730 (2006)

Andersson, T. and Biehl, A. "On the effective constitutive properties of a thin adhesive layer loaded in peel," International Journal of Fracture, 141, 227-246 (2006).

Andersson, T. and Stigh, U. "The stress-elongation relation for an adhesive layer loaded in peel using equilibrium of energetic forces," International Journal of Solids and Structures, 41, 413-434 (2004).

Chandra, N., Li, H., Shet, C. and H. Ghonem, H. "Some issues in the application of cohesive zone models for metal-ceramic interface," International Journal of Solids and Structures, 39, 2827-2855 (2002).

$\mathrm{Du}, \mathrm{J}$, Thouless, M. D. and Yee A. F. "Effects of rate on crack growth in a rubbermodified epoxy," Acta Mater., 48, 3581-3592 (2000).

Ferracin, T., Landis, C. M., Delannay, F. and Pardoen, T. "On the determination of the cohesive zone properties of an adhesive layer from the analysis of the wedge-peel test." International Journal of Solids and Structures, 40, 2889-2904 (2003).

Fleck, N. A., Hutchinson, J. W. and Suo Z. "Crack path selection in a brittle adhesive layer," International Journal of Solids and Structures, 27, 1683-1703 (1991).

Högberg, J. L., Sørensen, B. F. and Stigh, U. "Constitutive behaviour of mixed mode loaded adhesive layer," International Journal of Solids and Structures, 44, 83358354 (2007).

Li, H. and Chandra, N. "Analysis of crack growth and crack-tip plasticity in ductile materials using cohesive zone models," International Journal of Plasticity, 19, 849882 (2003). 
Li S., Thouless, M. D., Waas, A. M., Schroeder, J. A. and Zavattieri, P. D. "Use of a cohesive-zone model to analyze the fracture of a fiber-reinforced polymer-matrix composite," Composites Science and Technology, 65, 537-549 (2005b).

Li, V. C., Chan, C. M. and Leung, C. K. Y. "Experimental determination of the tensionsoftening relations for cementitious composites," Cement and Concrete Research, 17, 441-52 (1987).

Madhusudhana, K. S. and Narasimhan, R. "Experimental and numerical investigations of mixed-mode crack growth resistance of a ductile adhesive joint," Engineering Fracture Mechanics, 69, 865-883 (2002).

Parmigiani, J. P. and Thouless, M. D. "The Effects of Cohesive Strength and Toughness on Mixed-mode Delamination of Beam-Like Geometries," Engineering Fracture Mechanics, 74, 2675-2699 (2007).

Sørensen, B. F. "Cohesive law and notch sensitivity of adhesive joints," Acta Materialia, 50, 1053-61 (2002).

Sørensen, B. F. and Jacobsen, T. K. "Determination of cohesive laws by the $J$ integral approach," Engineering Fracture Mechanics, 70, 1841-185 (2003).

Sørensen, B. F. and Kirkgaard, P. "Determination of mixed-mode cohesive laws," Engineering Fracture Mechanics, 2642-2661 (2006).

Su, C., Wei, Y.J. and Anand, L. "An elastic-plastic interface constitutive model: application to adhesive joints," International Journal of Plasticity, 20, 2063-2081 (2004).

Sun, C. "Fracture of Plastic-deforming, Adhesively-bonded Structure: Experimental and Numerical Studies," Ph.D. dissertation, University of Michigan, Ann Arbor, MI (2007).

Sun, C., Thouless, M. D., Waas, A. M., Schroeder, J. A. and Zavattieri, P. D. "Ductilebrittle transitions in the fracture of plastically-deforming, adhesively-bonded 
structures: I Experimental studies," International Journal of Solids and Structures, 45, 3059-3073 (2008a).

Sun, C., Thouless, M. D., Waas, A. M., Schroeder, J. A. and Zavattieri, P. D. "Ductilebrittle transitions in the fracture of plastically-deforming, adhesively-bonded structures: II Numerical studies," International Journal of Solids and Structures, in press $(2008 b)$.

Sun, C., Thouless, M. D., Waas, A. M., Schroeder, J. A. and Zavattieri, P. D. "Rate effects in mode-II fracture of plastically-deforming, adhesively-bonded structures," under review (2008c)

Tvergaard, V. and Hutchinson, J. W. "Toughness of an interface along a thin ductile layer joining elastic solids" Philosophical Magazine A, 70, 641-656 (1994).

Tvergaard, V. and Hutchinson, J. W. "On the toughness of ductile adhesive joints," Journal of the Mechanics and Physics of Solids, 44, 789-800 (1996).

Ungsuwarungsri, T. and Knauss, W. G. "Role of damage-softened material behavior in the fracture of composites and adhesives," International Journal of Fracture, 35, 221-41 (1987).

Yang, Q. D., Thouless, M. D. and Ward, S. M. "Numerical simulations of adhesively bonded beams failing with extensive plastic deformation," Journal of the Mechanics and Physics of Solids, 47, 1337-1353 (1999).

Yang.Q. D., and Thouless, M. D. "Mixed-mode fracture analyses of plasticallydeforming adhesive joints," International Journal of Fracture, 110, 175-187 (2001). 
Table 1 Cohesive parameters

\begin{tabular}{|c|c|c|c|c|c|}
\hline \multicolumn{2}{|c|}{$\begin{array}{l}\text { Failure } \\
\text { mode }\end{array}$} & $\begin{array}{c}\text { Crack } \\
\text { propagation }\end{array}$ & $\begin{array}{c}\text { Crack } \\
\text { velocity } \\
(\mathrm{mm} / \mathrm{s})\end{array}$ & $\begin{array}{l}\text { Toughness } \\
\left(\mathbf{k J} / \mathbf{m}^{2}\right)\end{array}$ & $\begin{array}{c}\text { Cohesive } \\
\text { strength (MPa) }\end{array}$ \\
\hline \multirow{2}{*}{ 葛 } & \multirow{2}{*}{$\frac{7}{d}$} & Quasi-static & 0.07 1000 & $\Gamma_{\mathrm{I}}=3.9 \sim 4.5$ & \multirow[t]{2}{*}{$\hat{\sigma}=\mathbf{2 1} \sim 27$} \\
\hline & & Dynamic & -- & $\Gamma_{\mathrm{I}}^{\mathrm{d}}=1.0 \sim 1.1$ & \\
\hline \multirow{6}{*}{ 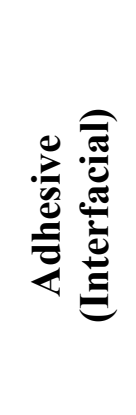 } & \multirow{2}{*}{$\frac{7}{d}$} & Quasi-static & $0.8 \sim 2200$ & $\Gamma_{\mathrm{Ii}}=2.5 \sim 3.1$ & \multirow{2}{*}{$\hat{\sigma}_{i}=21 \sim 25$} \\
\hline & & Dynamic & -- & $\Gamma_{I i}^{d}=0.51 \sim 0.65$ & \\
\hline & \multirow{4}{*}{$\frac{7}{\frac{1}{d}}$} & \multirow{4}{*}{ Quasi-static } & $0.2 \sim 0.6$ & $\Gamma_{\mathrm{II}}=8 \sim 16$ & $\hat{\tau}=20 \sim 26$ \\
\hline & & & 17 23 & $\Gamma_{\mathrm{II}}=12 \sim 23$ & $\hat{\tau}=23 \sim 27$ \\
\hline & & & $350 \sim 410$ & $\Gamma_{\mathrm{II}}=12 \sim 23$ & $\hat{\tau}=25 \sim 31$ \\
\hline & & & $300 \sim 2400$ & $\Gamma_{\mathrm{II}}=12 \sim 23$ & $\hat{\tau}=25 \sim 50$ \\
\hline
\end{tabular}




\section{Figure captions}

Figure 1 Configuration of (a) asymmetric tensile specimen, and (b) asymmetric double-cantilever-beam specimen. The out-of-plane width of both specimens is $25 \mathrm{~mm}$.

Figure 2 Micrograph of experimental setup and deformed specimen for an ADCB test. A high-resolution $\mathrm{CCD}$ camera was used to monitor the crack propagation. The beam was pre-painted white to help determine the location of the crack tip more accurately.

Figure 3 Comparison of load versus cross-head displacement plots for tests that exhibited "stick-slip" behavior and typical plots for the tests that exhibited quasi-static behavior.

Figure 4 Comparison of crack extension versus cross-head displacement plots for tests that exhibited "stick-slip" behavior and typical plots for the tests that exhibited quasi-static behavior.

Figure 5 Optical micrograph of a fracture surface of an ADCB specimen that exhibited both quasi-static growth and dynamic growth.

Figure 6 A comparison between the experimental results for the asymmetric double-cantilever beam and the numerical predictions using the mode-I and mode-II values for the cohesive properties.

Figure 7 A comparison between the experimental results for the asymmetric double-cantilever beam and the numerical predictions for the crack length using the mode-I and mode-II values for the cohesive properties.

Figure 8 a plot of the range of cohesive parameters that resulted in an acceptable level of agreement between the numerical and experimental results. 
Figure 9 Geometry of the asymmetric wedge specimens. The width of specimen is $20 \mathrm{~mm}$.

Figure 10 Photograph of the experimental configuration for the drop tower tests with asymmetric wedge tests. A high-resolution CCD camera was used to monitor the crack propagation. The beam was pre-painted white to help determine the location of the crack tip more accurately

Figure 11 Plot of wedge displacement against time for different drop height. The uncertainties for measurements of the wedge displacement are $\pm 0.5 \mathrm{~mm}$.

Figure 12 Plot of crack extension against time for different drop heights. The uncertainties for measurements of the crack length are $\pm 1 \mathrm{~mm}$.

Figure 13 Plot of crack length ahead of the wedge tip against crack extension for both the tests that exhibited only quasi-static crack growth and the tests that exhibited some regions of dynamic fracture. The uncertainties for measurements of the crack length are $\pm 1 \mathrm{~mm}$.

Figure 14 The static ABAQUS/Standard 2D model used to simulate the asymmetric wedge tests. Contact elements were placed along the surfaces of the wedge and adherend.

Figure 15 Configuration of lap shear test specimen. The width of specimen is $20 \mathrm{~mm}$.

Figure 16 A comparison between the numerical predictions for the loaddisplacement curves for the single-lap shear test and the experimental results. 


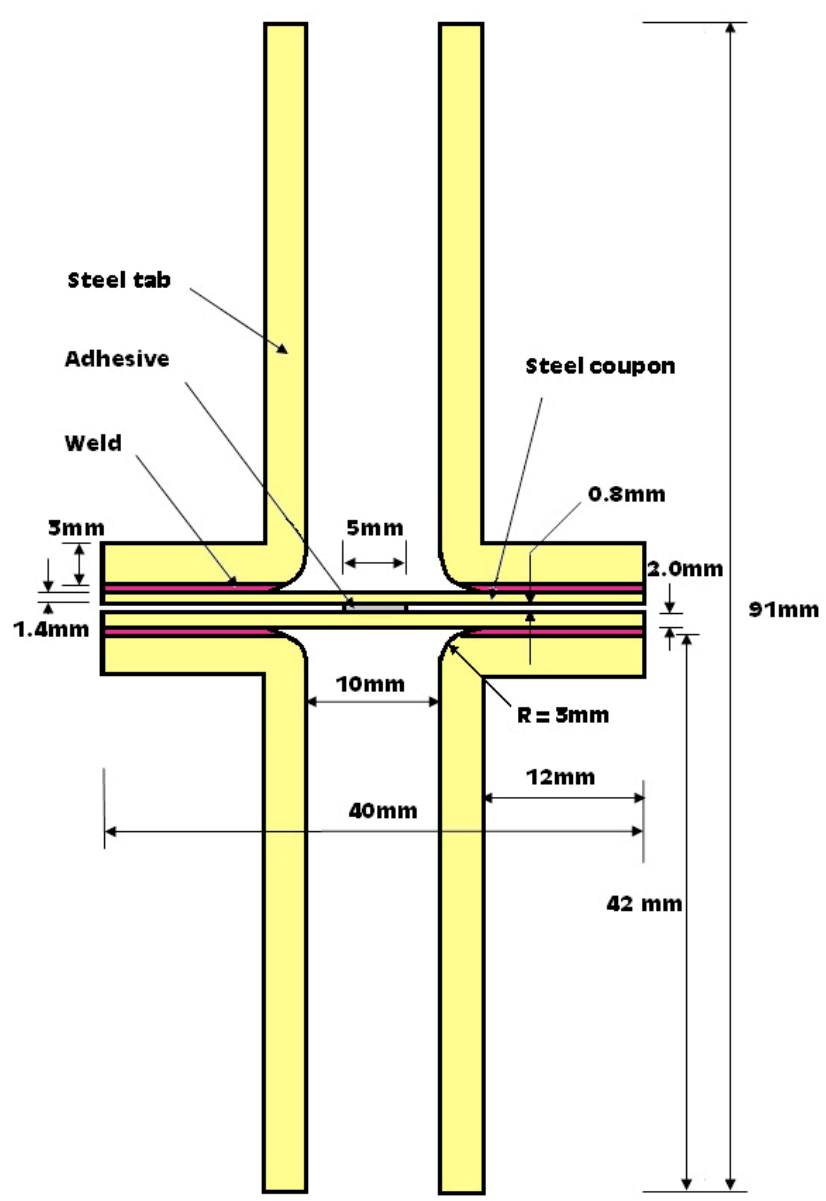

(a)

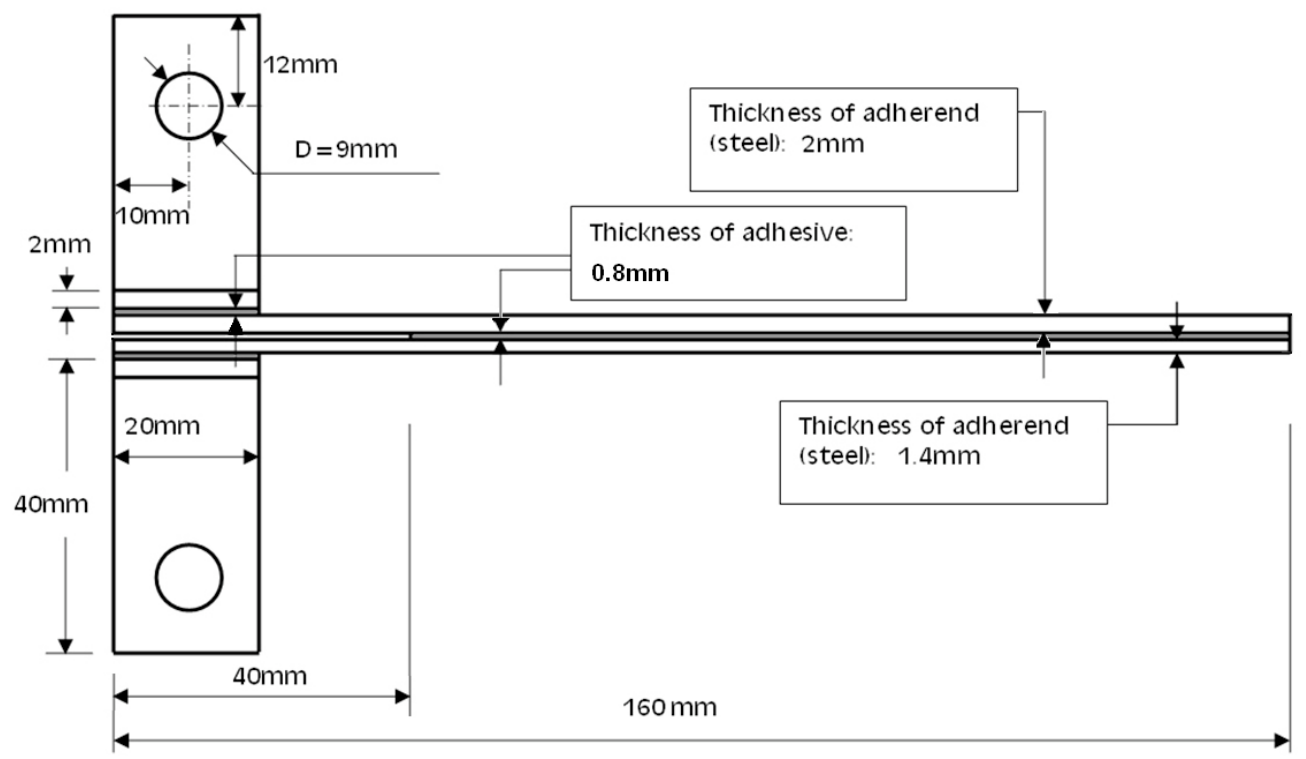

(b)

Figure 1 Configuration of (a) asymmetric tensile specimen, and (b) asymmetric double-cantilever-beam specimen. The out-of-plane width of both specimens is $25 \mathrm{~mm}$. 


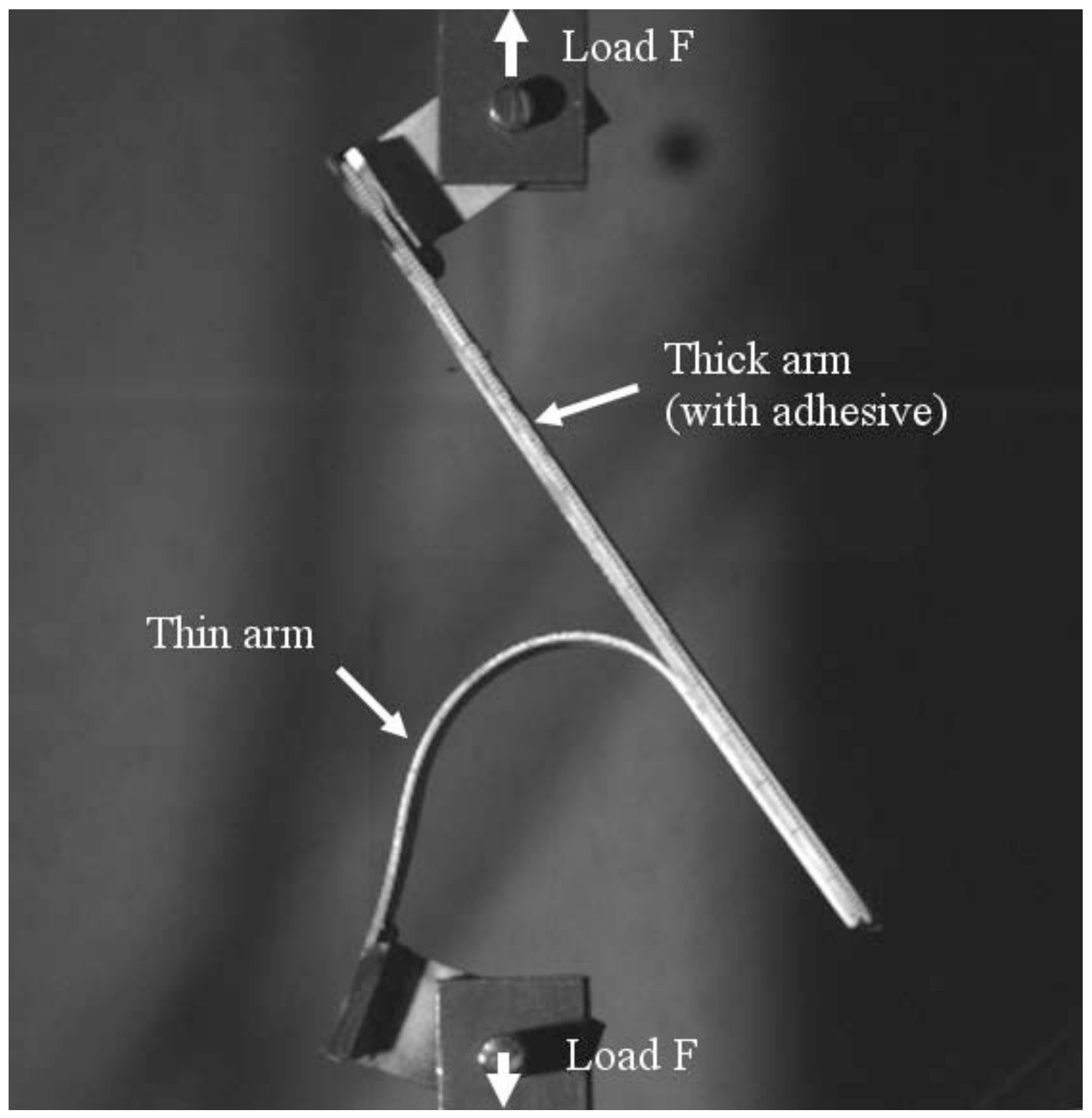

Figure 2 Micrograph of experimental setup and deformed specimen for an ADCB test. A high-resolution $\mathrm{CCD}$ camera was used to monitor the crack propagation. The beam was pre-painted white to help determine the location of the crack tip more accurately. 


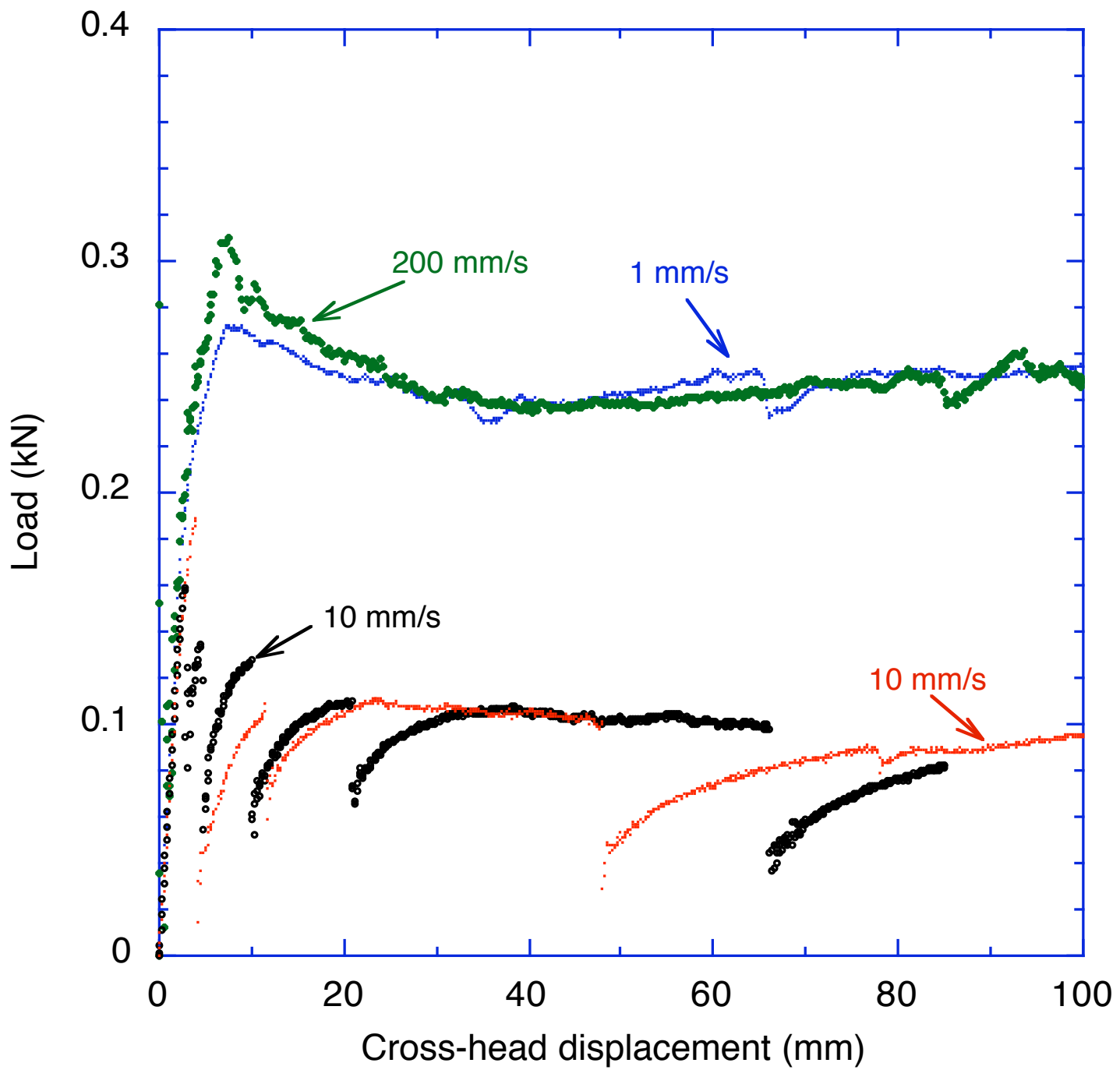

Figure 3 Typical examples for the plots of the load against cross-head displacement for asymmetrical DCB tests, showing both quasi-static behavior and transitions to dynamic fracture. 


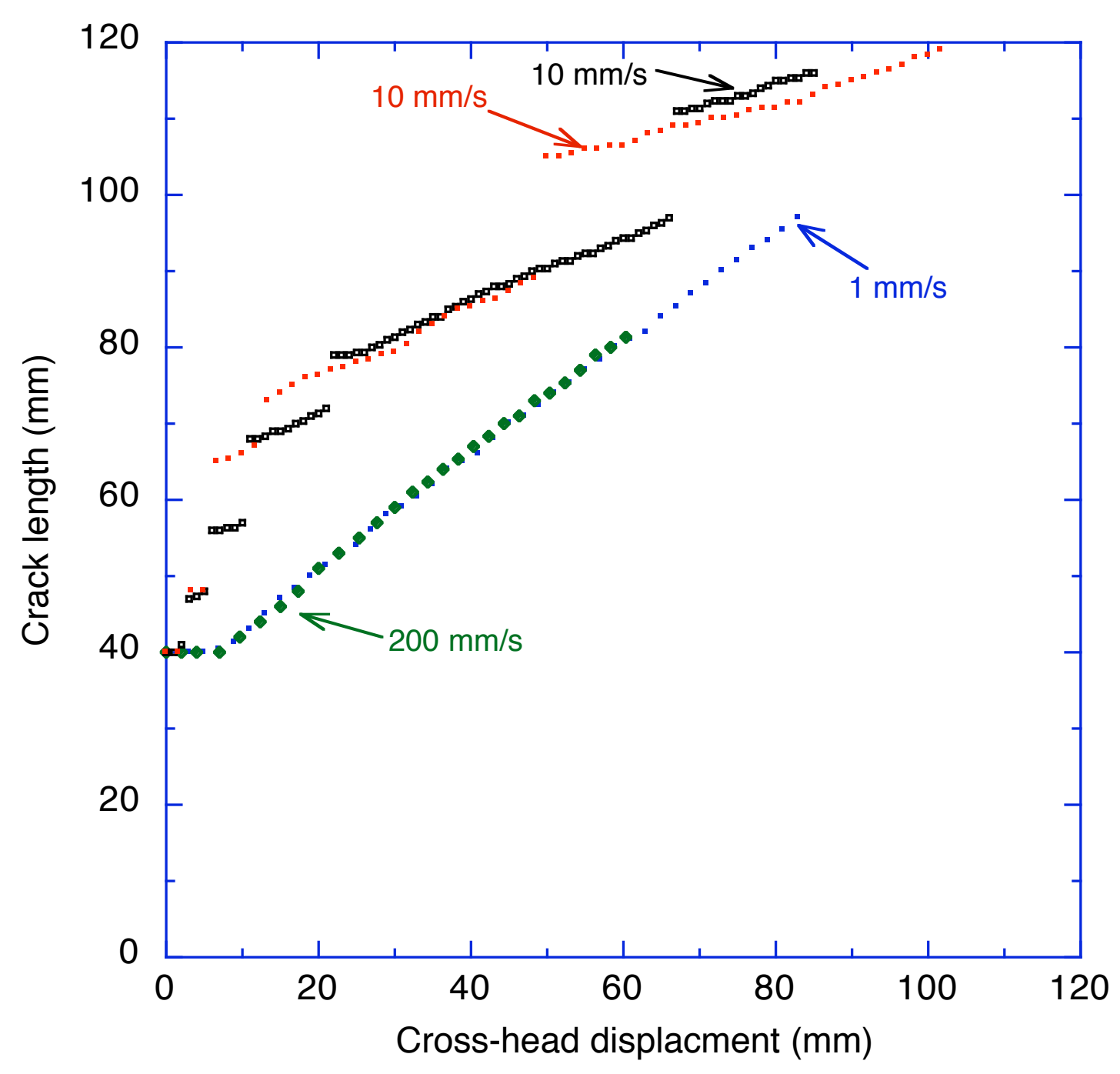

Figure 4 Typical examples for the plots of the crack length against cross-head displacement for asymmetrical DCB tests, showing both quasi-static behavior and transitions to dynamic fracture. 


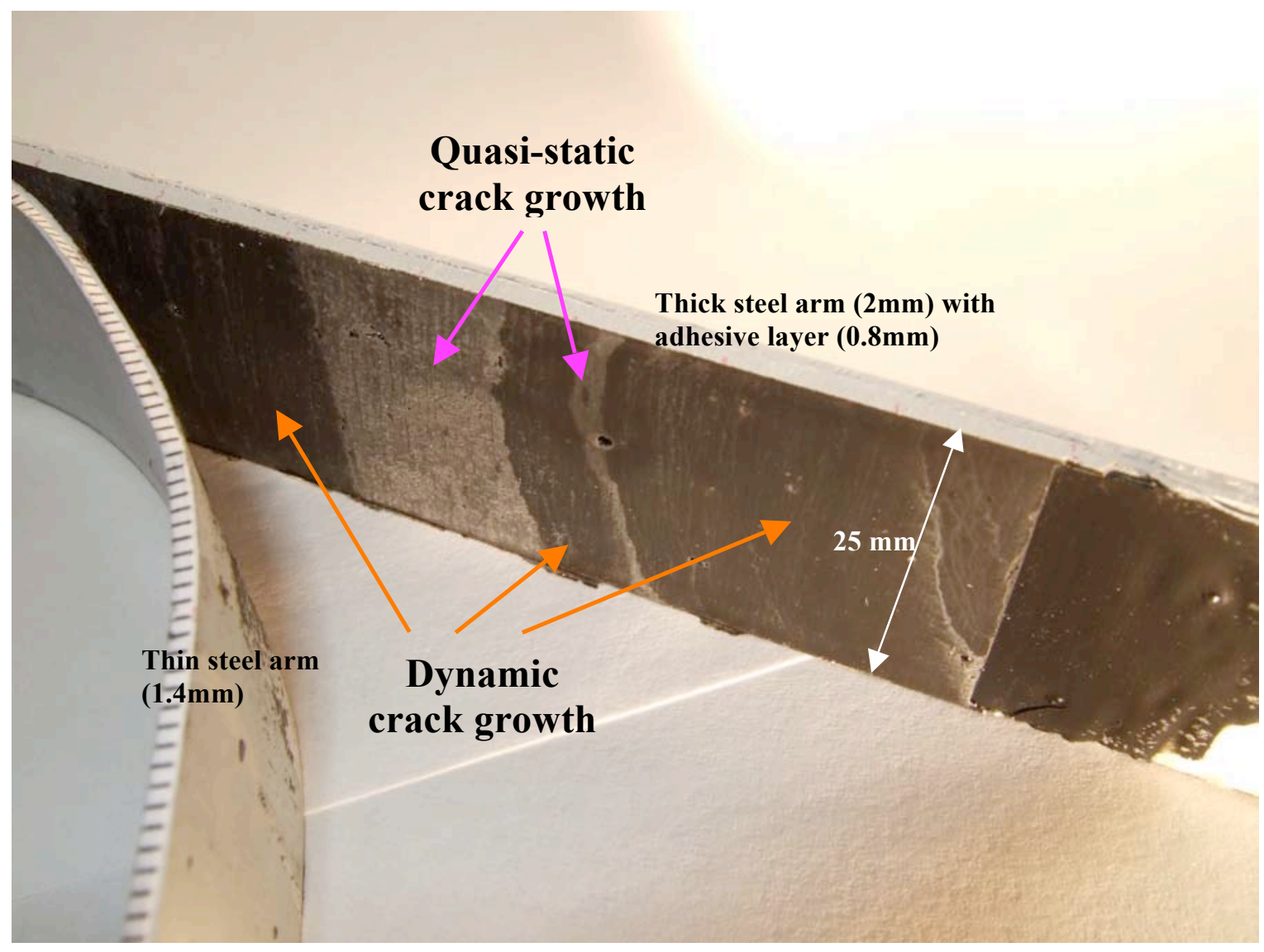

Figure 5 Optical micrograph of a fracture surface of an ADCB specimen that exhibited both quasi-static growth and dynamic growth. 


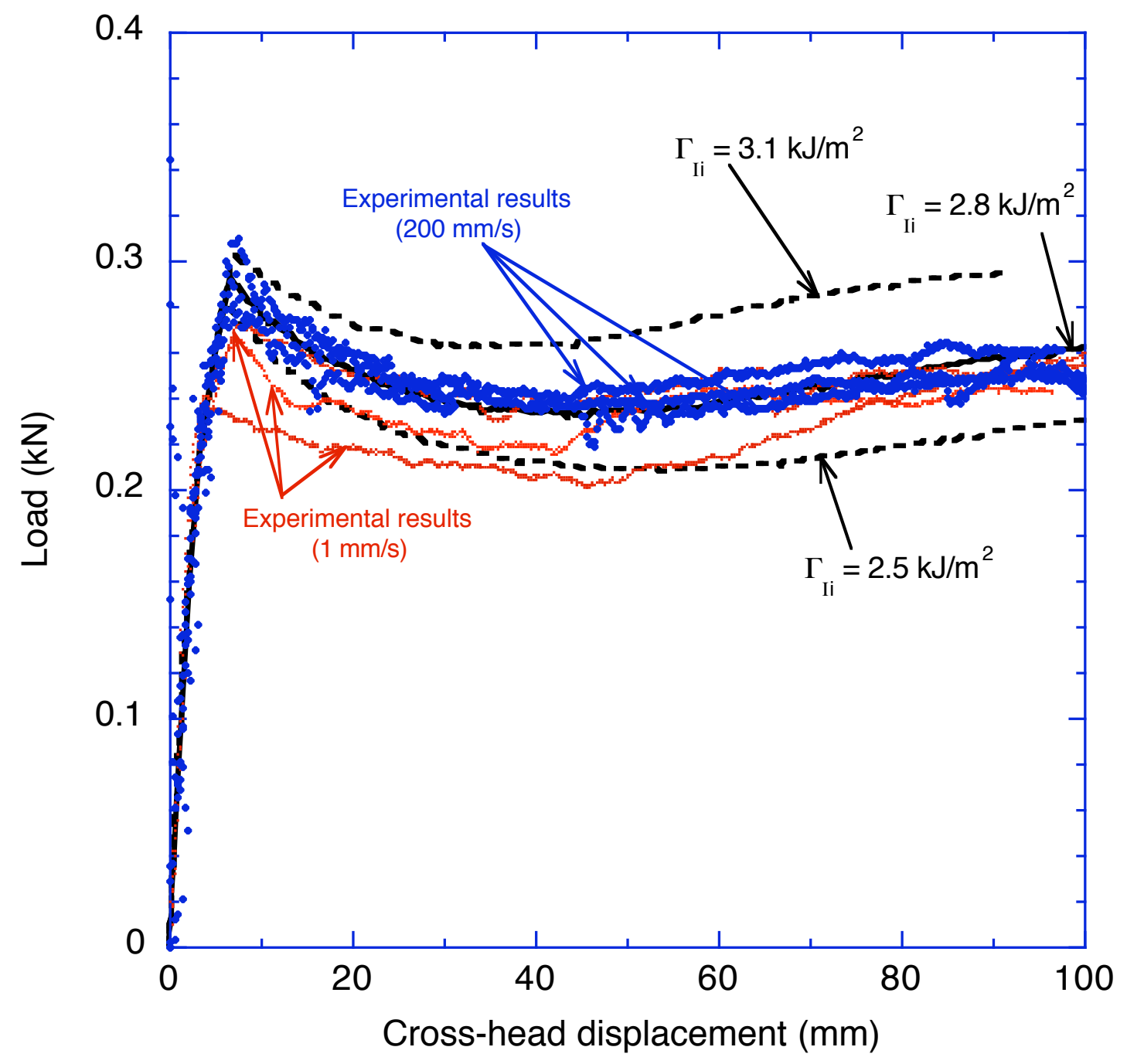

Figure 6 A comparison between the experimental results for the asymmetrical double-cantilever beam, and the numerical results used to determine the mode-I interfacial toughness. 


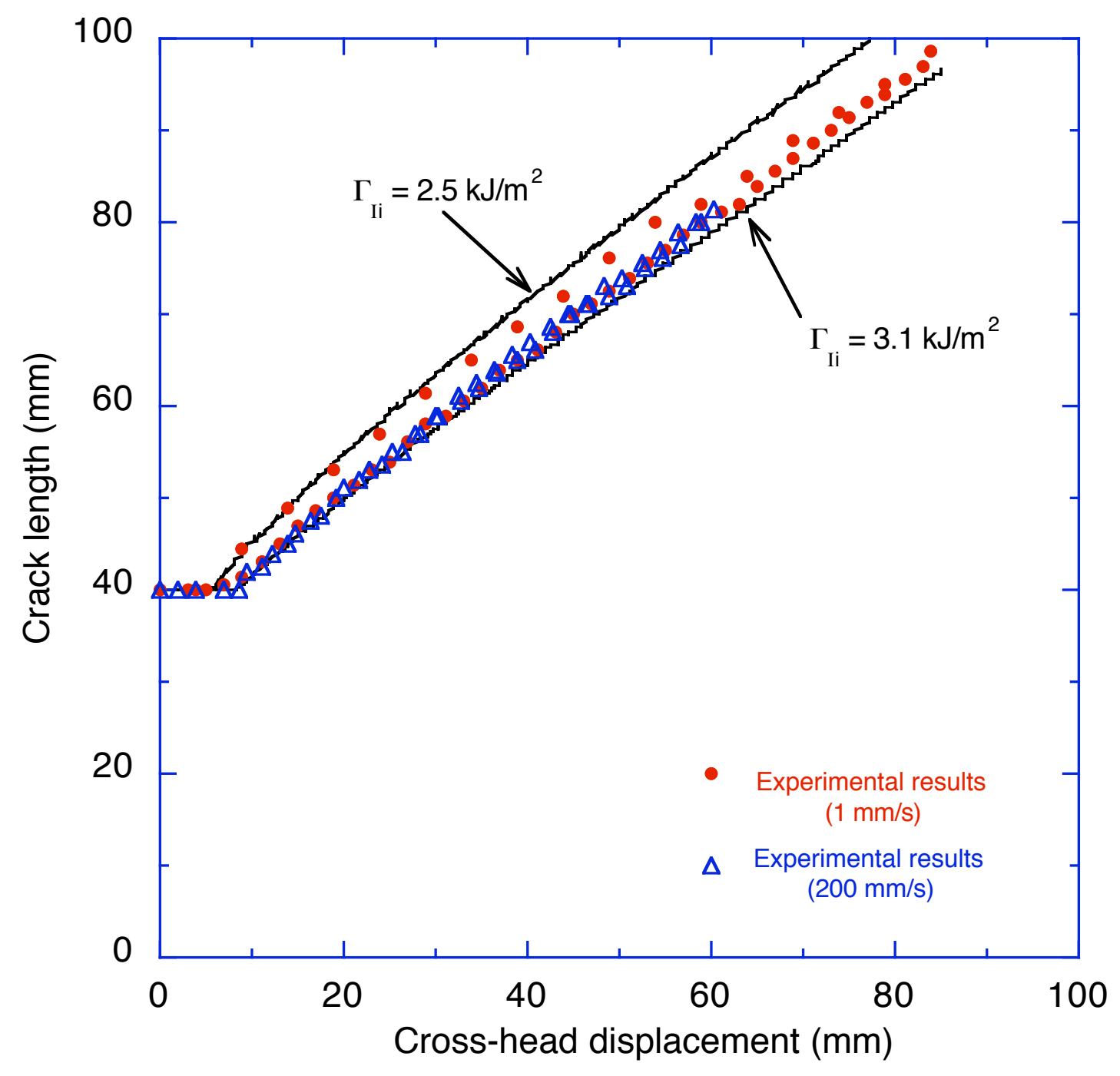

Figure 7 A comparison between the experimental results for the asymmetrical double-cantilever beam and the numerical predictions for the crack length.. 


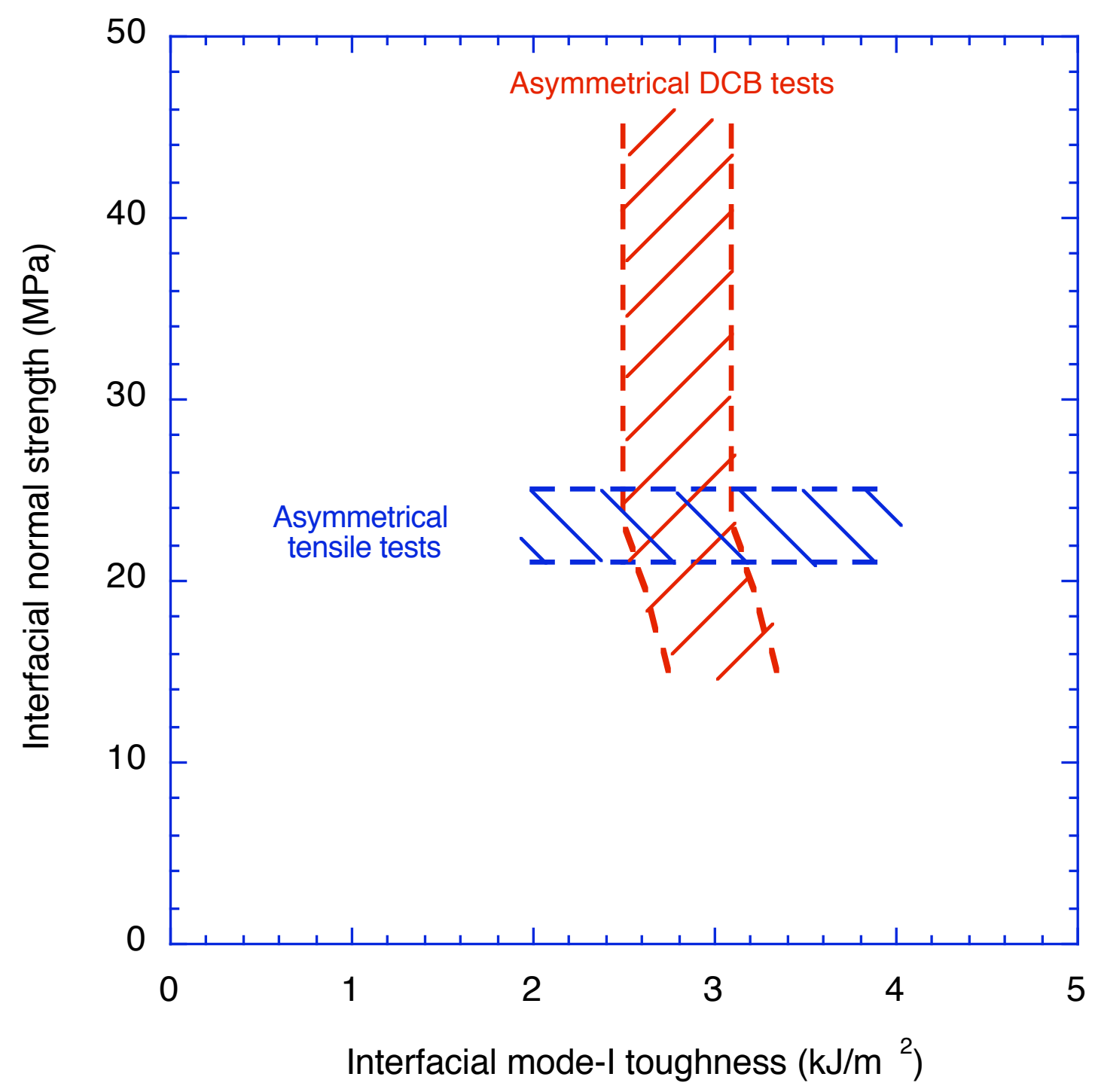

Figure 8 A plot of the range of interfacial cohesive parameters that resulted in acceptable levels of agreement between the numerical and experimental results for the asymmetrical DCB and tensile tests. 


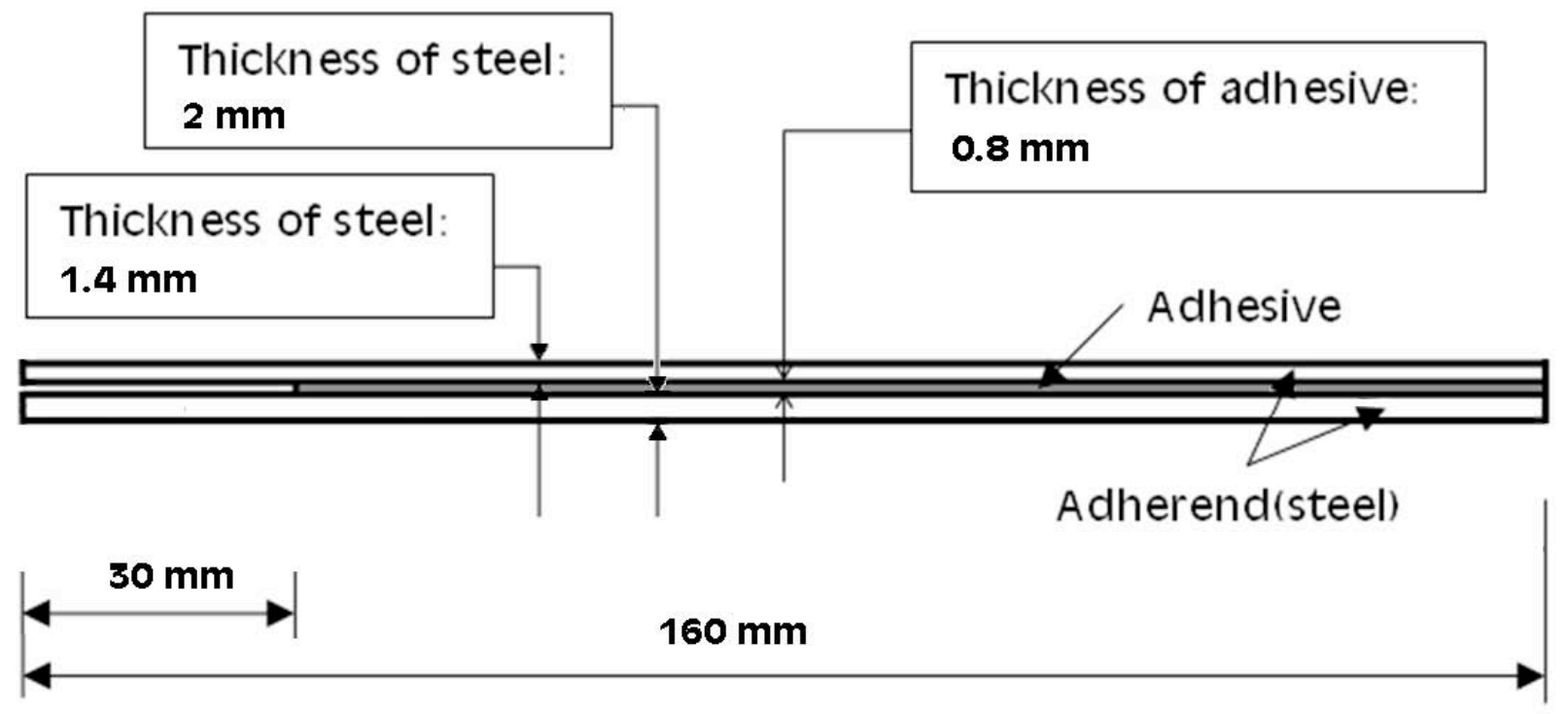

Figure 9 Geometry of the asymmetric wedge specimens. The width of specimen is $20 \mathrm{~mm}$. 


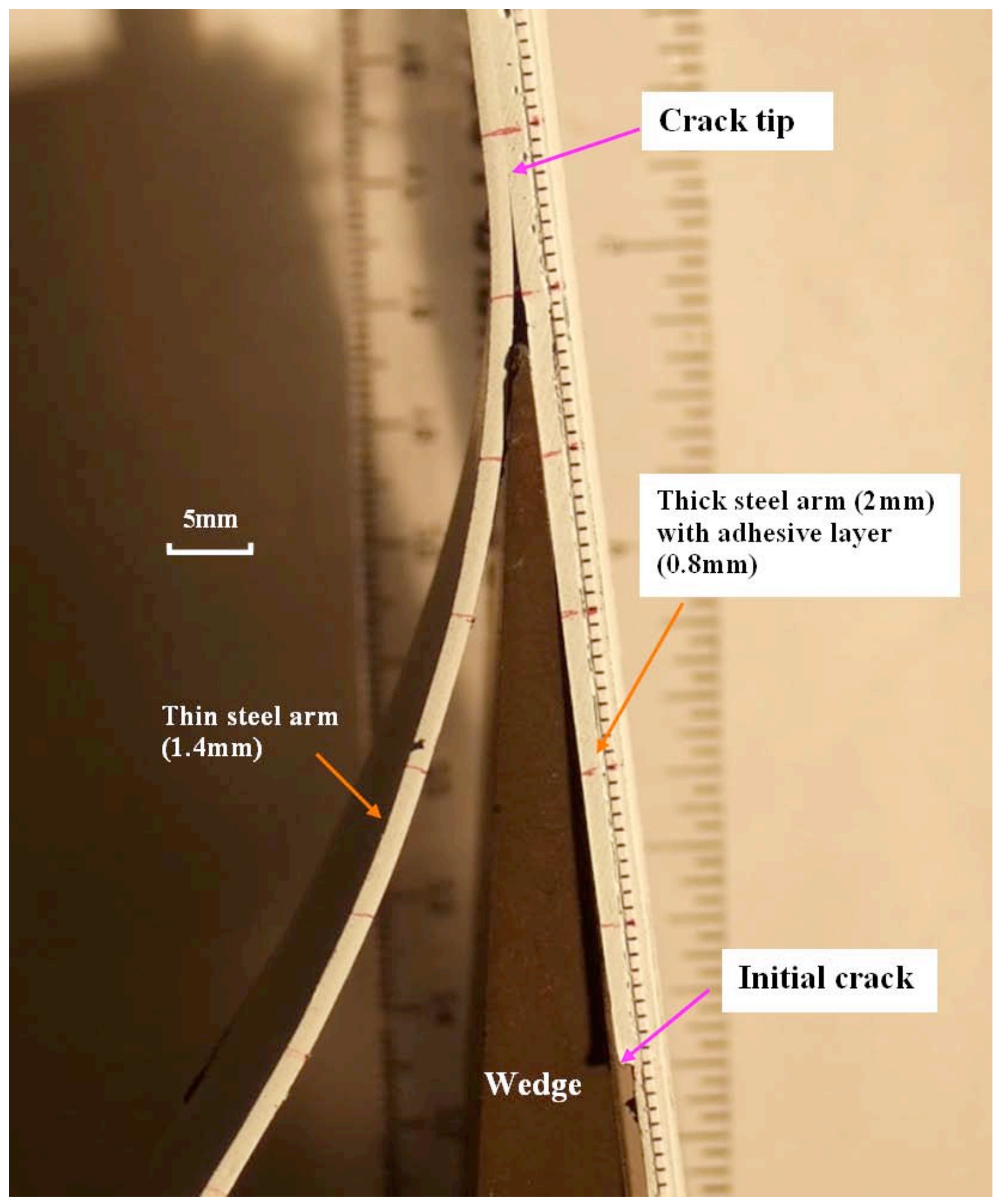

Figure 10 Photograph of the asymmetrical wedge tests. A high-resolution CCD camera was used to monitor the crack propagation. The sides of the specimen were painted white to help determine the location of the crack tip more accurately. 


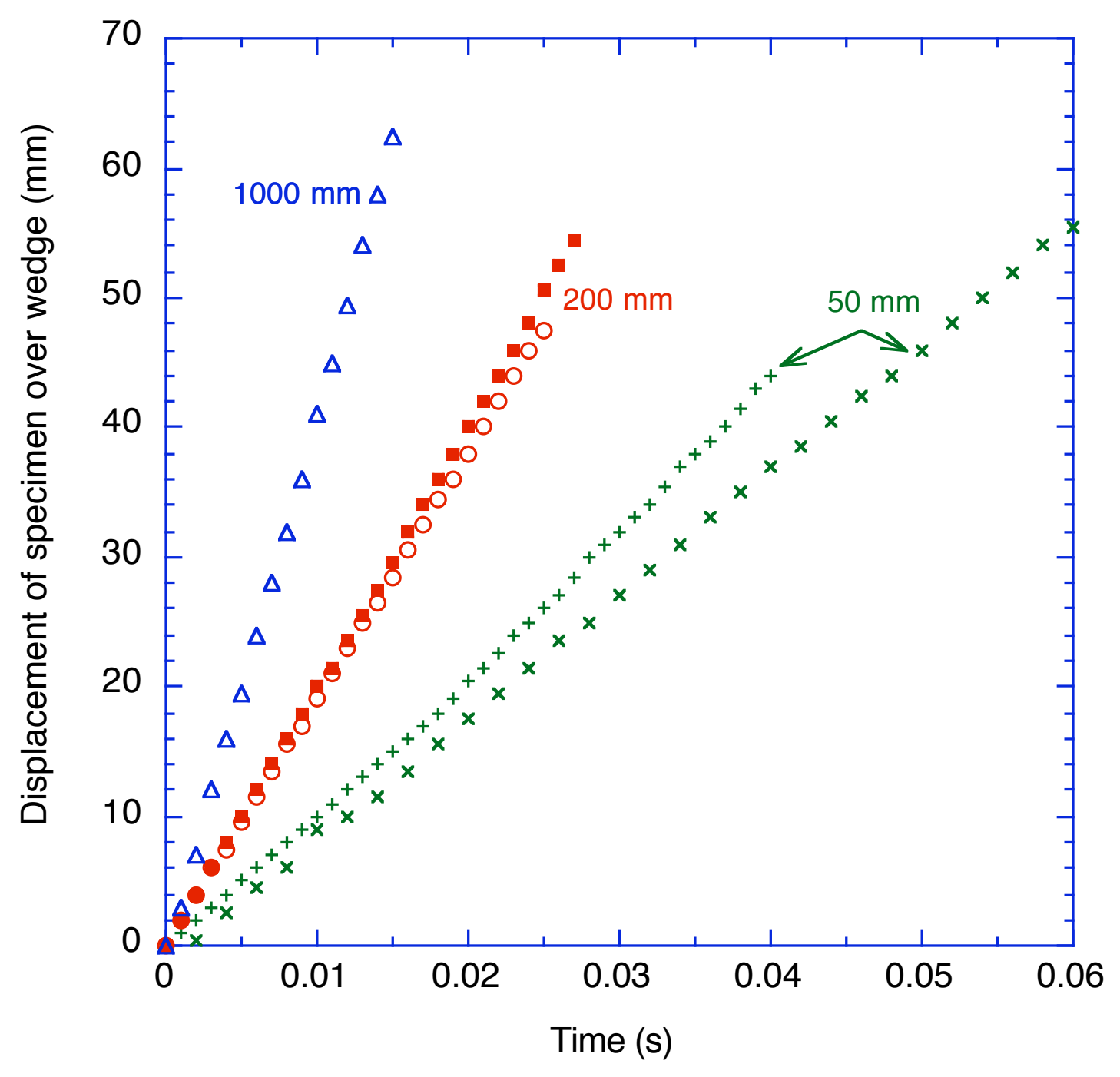

Figure 11 Plots of the displacement of the specimen relative to the wedge against time for different drop heights. The uncertainties for measurements of the displacement are $\pm 0.5 \mathrm{~mm}$. 


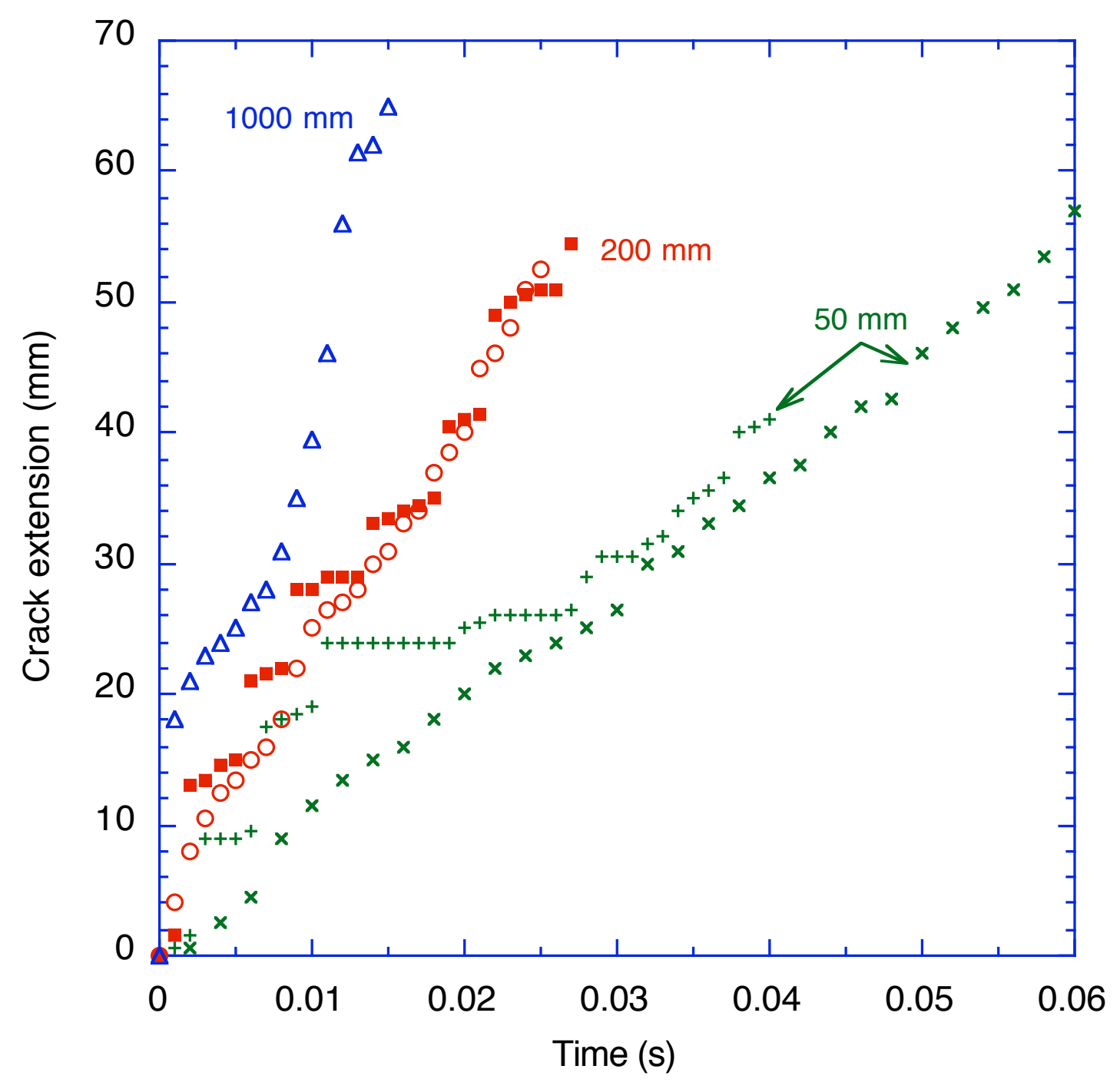

Figure 12 Plot of crack extension against time for different drop heights. The uncertainties for measurements of the crack length are $\pm 1 \mathrm{~mm}$. 


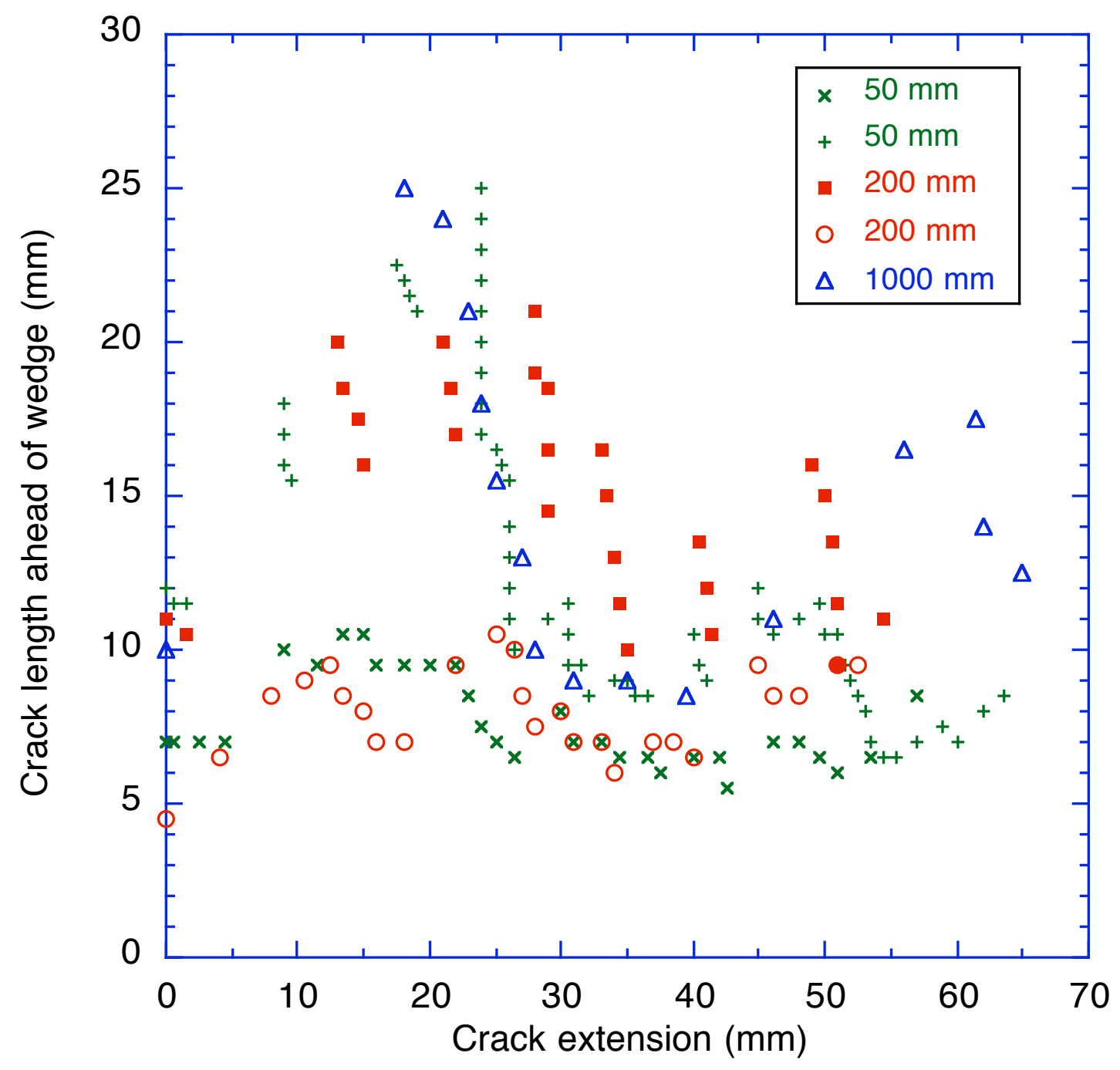

Figure 13 Plot of crack length ahead of the wedge tip against crack extension for both the tests that exhibited only quasi-static crack growth and the tests that exhibited some regions of dynamic fracture. The uncertainties for measurements of the crack length are $\pm 1 \mathrm{~mm}$. 


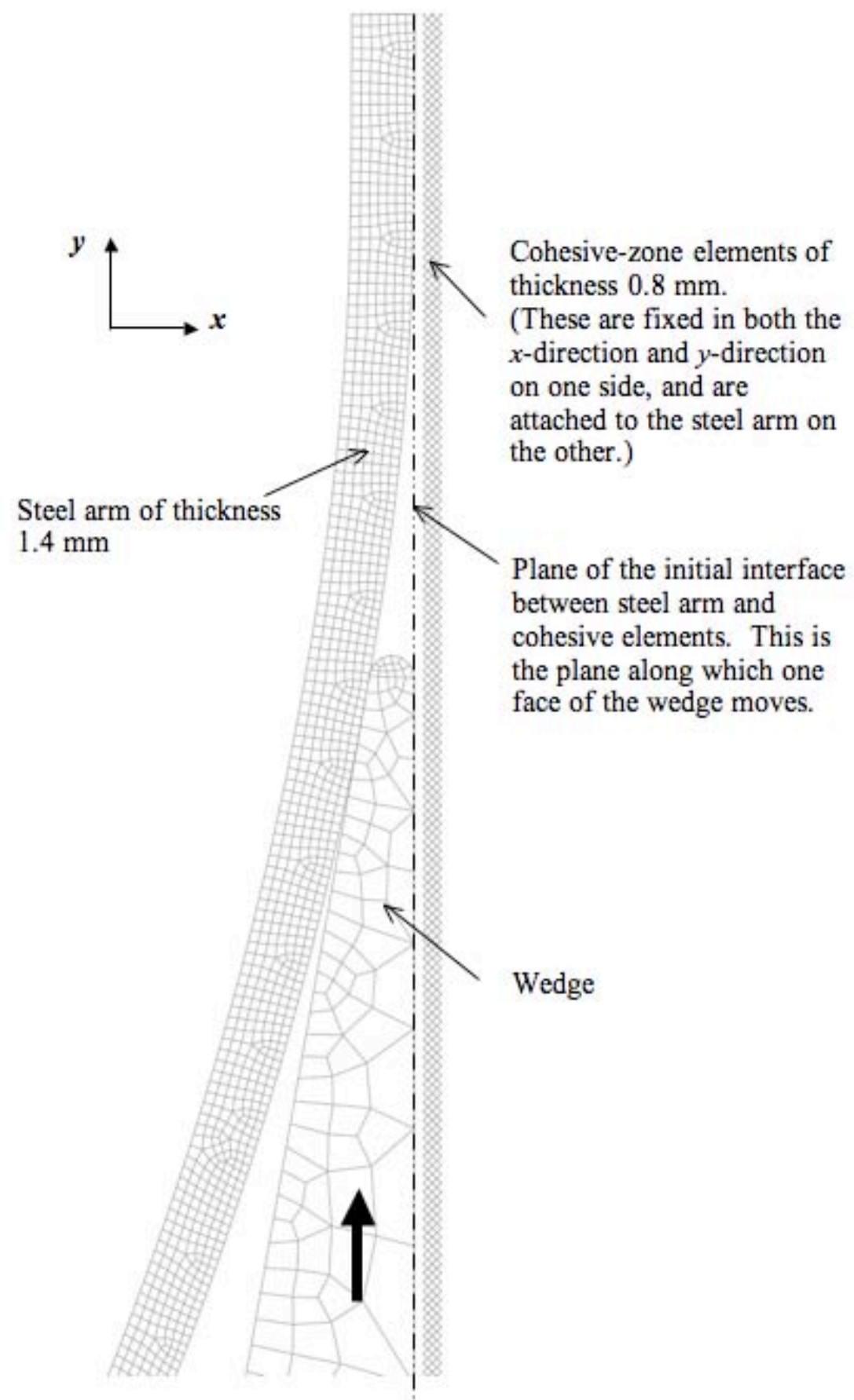

Figure 14 The static ABAQUS/Standard 2D model used to simulate the asymmetric wedge tests. Contact elements were placed along the surfaces of the wedge and adherend. 


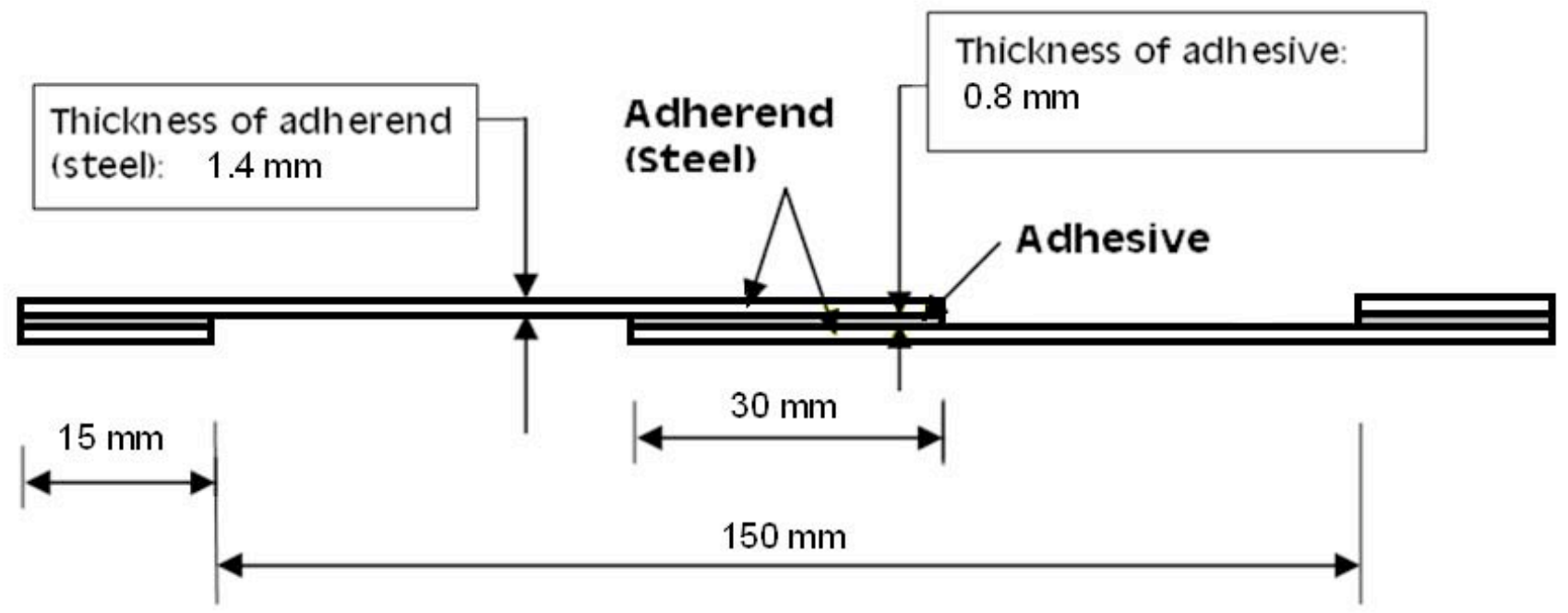

Figure 15 Configuration of lap shear test specimen. The width of specimen is $20 \mathrm{~mm}$. 


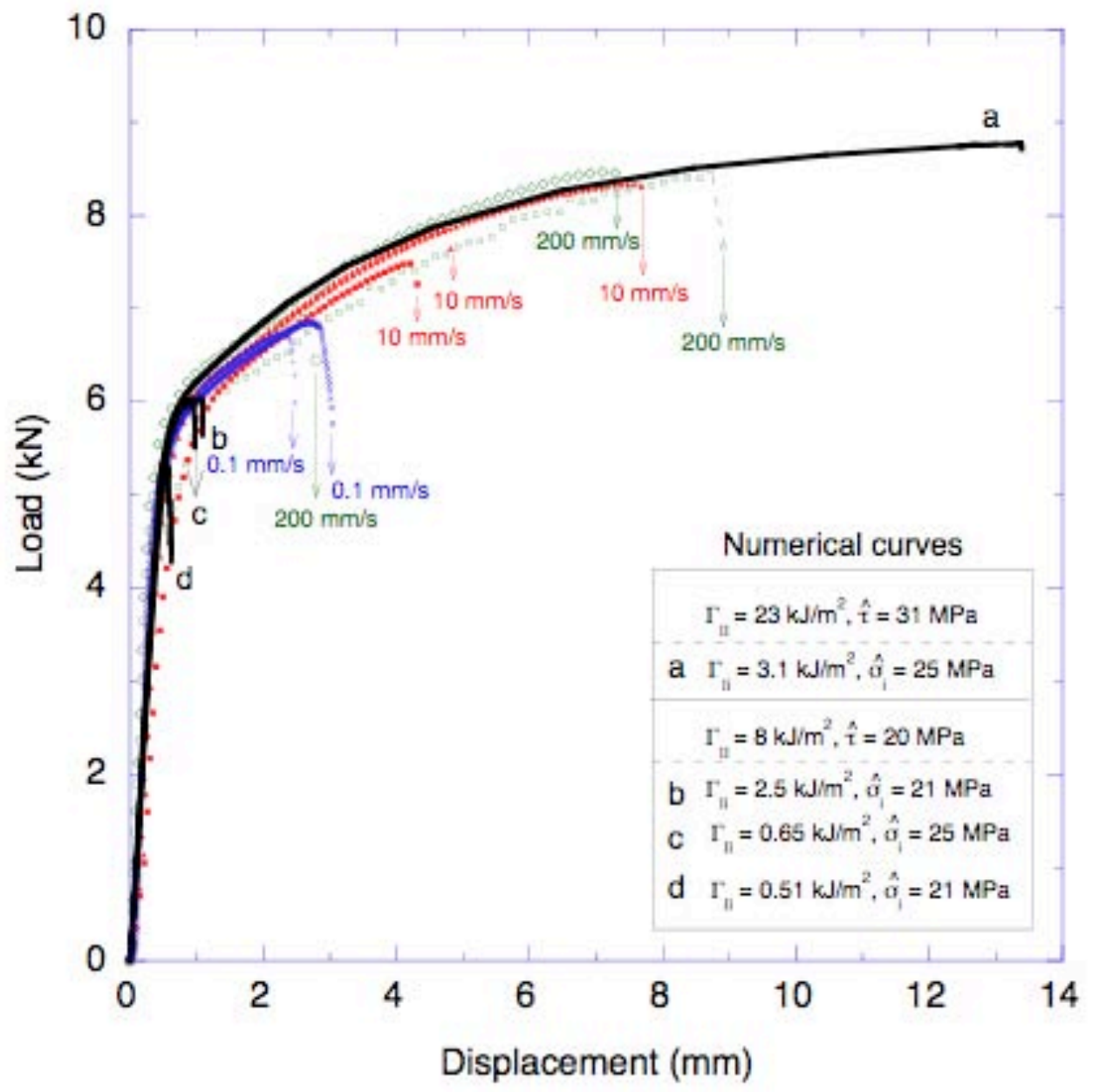

Figure 16 A comparison between the numerical predictions for the loaddisplacement curves for the single-lap shear test and the experimental results. 